ARTICLE

httpst//doi.org/10.1038/s41467-022-28253-4

\title{
Splicing is an alternate oncogenic pathway activation mechanism in glioma
}

\author{
Robert Siddaway (10 1, Scott Milos ${ }^{1}$, Arun Kumaran Anguraj Vadivel (1) 1, Tara H. W. Dobson², \\ Jyothishmathi Swaminathan ${ }^{2}$, Scott Ryall', Sanja Pajovic ${ }^{1}$, Palak G. Patel ${ }^{1}$, Javad Nazarian ${ }^{3,4}$, Oren Becher (1) ${ }^{5}$, \\ Michael Brudno (i] 6,7,8, Arun Ramani ${ }^{7}$, Vidya Gopalakrishnan (D) ${ }^{2}$ \& Cynthia Hawkins (i) 1,9,10凶
}

High-grade diffuse glioma ( $H G G$ ) is the leading cause of brain tumour death. While the genetic drivers of HGG have been well described, targeting these has thus far had little impact on survival suggesting other mechanisms are at play. Here we interrogate the alternative splicing landscape of pediatric and adult HGG through multi-omic analyses, uncovering an increased splicing burden compared with normal brain. The rate of recurrent alternative splicing in cancer drivers exceeds their mutation rate, a pattern that is recapitulated in pan-cancer analyses, and is associated with worse prognosis in HGG. We investigate potential oncogenicity by interrogating cancer pathways affected by alternative splicing in HGG; spliced cancer drivers include members of the RAS/MAPK pathway. RAS suppressor neurofibromin 1 is differentially spliced to a less active isoform in $>80 \%$ of HGG downstream from REST upregulation, activating the RAS/MAPK pathway and reducing glioblastoma patient survival. Overall, our results identify non-mutagenic mechanisms by which cancers activate oncogenic pathways which need to accounted for in personalized medicine approaches.

\footnotetext{
${ }^{1}$ Arthur and Sonia Labatt Brain Tumour Research Centre, The Hospital for Sick Children, Toronto, Canada. ${ }^{2}$ Department of Pediatrics - Research, The University of Texas MD Anderson Cancer Center, Houston, TX 77030, USA. ${ }^{3}$ Department of Integrative Systems Biology, Children's National Medical Center, George Washington University, Washington, DC 20010, USA. ${ }^{4}$ Department of Oncology, University Children's Hospital, Zurich, Switzerland. ${ }^{5}$ Ann \& Robert H. Lurie Children's Hospital of Chicago, Chicago, IL 60611, USA. ${ }^{6}$ Department of Computer Science, University of Toronto, Toronto, Canada. ${ }^{7}$ Centre for Computational Medicine, The Hospital for Sick Children, Toronto, Canada. ${ }^{8}$ University Health Network, Toronto, ON, Canada. ${ }^{9}$ Department of Laboratory Medicine and Pathobiology, University of Toronto, Toronto, Canada. ${ }^{10}$ Division of Pathology, The Hospital for Sick Children, Toronto, Canada.

凶email: cynthia.hawkins@sickkids.ca
} 
T he genetic drivers of high-grade glioma (HGG) have been well-described, with isocitrate dehydrogenase (IDH) mutations and epidermal growth-factor receptor (EGFR) amplifications common in adult diffuse glioma, while pediatric HGG (pHGG) harbor high-frequency mutations in histones H3.1 and $\mathrm{H} 3.3$ as well as platelet-derived growth-factor receptor alpha $(P D G F R A)$ alterations ${ }^{1-8}$. Some events are shared between pediatric and adult HGG; for example, neurofibromin 1 (NF1) is mutated in $5 \%$ of all cancers and $10 \%$ of $\mathrm{HGG}^{3,4,9}$ However, the prognosis for both children and adults diagnosed with this devastating disease remains dismal. The oncogenicity of DNA mutations has been well studied but is imperfect in its ability to predict tumor behavior and therapeutic response, suggesting other mechanisms, including epigenetic, transcriptomic, and proteomic, are at play. Alternative splicing (AS), which increases transcript diversity and has been shown to be altered in adult cancer ${ }^{10}$, has the potential to affect cancer genes through removal or alteration of protein domains and post-translational modifications (PTM), nonsense-mediated decay, or protein truncation.

Here, we find that AS more frequently targets cancer genes than do point mutations across a broad spectrum of cancers, a phenomenon that is particularly striking in HGG where cancer-driver splicing, but not mutation, burden is significantly associated with survival. Characterization of the HGG AS landscape demonstrates a convergence on cancer-driver genes. To test the potential for a functional role of these AS events, we investigate the role of alternative splicing of NF1. We found preferential inclusion of NF1 exon23a in HGG increased RAS/MAPK activity independent of other RAS/MAPK alterations and was associated with worse patient survival. $N F 1^{3,4,9}$, is differentially spliced in over $80 \%$ of $\mathrm{HGG}$, indicating a much broader, NF1-mediated, RAS-activating event than by mutation alone. Overall, our data demonstrate an alternative, clinically relevant, mechanism of oncogenic pathway activation in cancer that will be important to integrate into future therapeutic workflows for optimal precision medicine.

\section{Results}

Spliceosome mutations are associated with increased alternative splicing in pHGG. Investigating the mutational landscape of our pHGG cohort, we found that $34 \%$ of patients $(n=31 / 91)$ had at least one spliceosome mutation including single nucleotide variants or copy number alterations (Fig. 1a, Supplementary Fig. S1a, and Supplementary Data 1 and 2). In an independent pHGG cohort $^{11,12}, 28 \%(40 / 141)$ of patients had spliceosome mutations (Supplementary Fig. S1b). The spliceosome is composed of multiple subcomplexes, with many proteins involved in one or more subcomplexes (Supplementary Data 2). Core components that are used throughout the spliceosome lifecycle were the most frequently mutated, with $11-23 \%$ of patients $(n=10-21)$ having a mutation in any complex (Fig. 1a and Supplementary Fig. S1c). Spliceosome mutations in genes including $S F 3 B 1, U 2 A F$, and SRSF2 frequently occur in other tumors such as leukemia and myelodysplastic syndrome ${ }^{13}$, while highly recurrent alterations in the U1 snRNA were recently identified in multiple cancers, including medulloblastoma ${ }^{14,15}$. Hotspot mutations in these genes and the U1 snRNA alterations were not identified in our cohort (Supplementary Data 2).

Given the frequent spliceosome mutations, we hypothesized that pHGG may be prone to disrupted AS. In RNA-Seq analysis comparing 64 pHGG $(n=64)$ with the normal brain $(n=20)$, splicing pathways were upregulated (Fig. $1 \mathrm{~b}$ and Supplementary Data 1), irrespective of direct mutations in the spliceosome and not related to differential DNA methylation (Supplementary Fig. S1f). An upregulation of splicing pathways was also seen in adult diffuse glioma using RNA-Seq from The Cancer Genome Atlas (TCGA; IDH-wt $(n=243)$, IDH-mut/non-codel $(n=270)$, IDH-mut/codel $(n=173)$, normal brain $(n=5))$ and $(P \leq 0.005$; Supplementary Fig. S1d, e) suggesting this may be a general glioma phenomenon.

In keeping with the upregulation of splicing pathways, analysis of alternative splicing events (ASE) (rMATS ${ }^{16}$ ), found 247,507 ASE across the dataset (Supplementary Fig. S2a, b) with 1827 differential ASE from 904 genes identified in pHGG compared with a normal brain. 844 differential ASE (absolute $\Delta \psi>0.15$, FDR $<0.05)$ associated with mutant spliceosomes, which were mostly skipped exons or mutually exclusive exons and unique to tumors mutated in a given complex (Fig. 1e and Supplementary Fig. S1j, k). Subcomplexes E (the earliest assembly stage) and Bact (the subcomplex where the catalytic core is fully assembled) had the biggest effect on AS compared with spliceosome-WT tumors (Fig. 1a, e and Supplementary Fig. S1k).

Globally, pHGG and normal brain could be efficiently separated in unsupervised hierarchical clustering and t-SNE analyses based on ASEs (Supplementary Fig. S1g, h). We then quantified alternative splicing in pHGG compared with the normal brain. First, we found that splice junction usage was more variable $\left(P<2 \times 10^{-16}\right.$; Supplementary Fig. S1i). Next, we calculated the Shannon entropy of each multi-isoform gene ${ }^{17}$, finding a significant increase in pHGG indicating that more isoforms are expressed than in the normal brain, irrespective of spliceosome-mutant status (pHGG as a whole vs normal brain $P<2 \times 10^{-16}$; spliceosome-WT pHGG vs. normal brain $P=6 \times 10^{-11}$; spliceosome-mutant $\mathrm{PHGG}$ vs. normal brain $P<10^{-15}$; spliceosome-WT vs. spliceosome-mutant pHGG $P=0.037$, Fig. 1c, d).

Cancer-driver genes are enriched among differential splicing events in pHGG. In total, 1827 differential ASE from 904 genes were identified in pHGG, predominantly skipped exon and mutually exclusive exon events, (Fig. 2a and Supplementary Fig. $\mathrm{S} 2 \mathrm{a}-\mathrm{c}$ ). We validated select targets by RT-PCR in $\mathrm{pHGG/normal}$ brain pairs (Supplementary Fig. S2d). In all, 79\% of spliced genes were not also differentially expressed, indicating the differential ASE we identified are not expression-level artifacts (Supplementary Fig. S2e). To confirm our findings, we compared the targets identified by rMATS $^{16}$ with a second algorithm, SUPPA $2^{18}$, which estimates splicing changes from transcript abundances rather than junction counts and thus provides a very different method of analyzing the splicing landscape of pHGG. SUPPA2 identified 2576 events from 1482 genes, with a highly significant overlap in differentially spliced genes between the two approaches $\left(P=10^{-120}\right.$; Supplementary Fig. S2f). Interestingly, although large numbers of intron retentions have recently been described in adult GBM${ }^{10,19}$, neither rMATS (6) or SUPPA2 (30) identified large numbers of significantly differential intron retentions when comparing $\mathrm{pHGG}$ with normal brain despite identifying over 4000 intron retention events across the dataset. This could be algorithm-related, however, as using SplAdder, the algorithm used in the adult GBM publication ${ }^{10}$, identified $>3 \times$ more $(13,866)$ RI events, and determined 565 to be significant (94 and $20 \times$ more than $\mathrm{rMATS} / \mathrm{SUPPA} 2$, respectively). Importantly, three genes in which we confirmed differential splicing in pHGG using RT-PCR (FGFR1, MBD1, SMARCC2) were identified only with rMATS. Given that rMATS could identify additional true splicing events beyond SUPPA2, while appearing more conservative overall in terms of events identified, we proceeded with the 1827 differential ASE we identified in pHGG using rMATS for subsequent analyses.

We found that the 1827 ASE in pHGG were differentially spliced regardless of the spliceosome mutation status. In addition, 
a

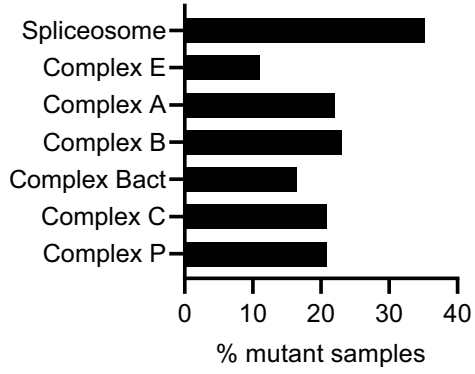

b

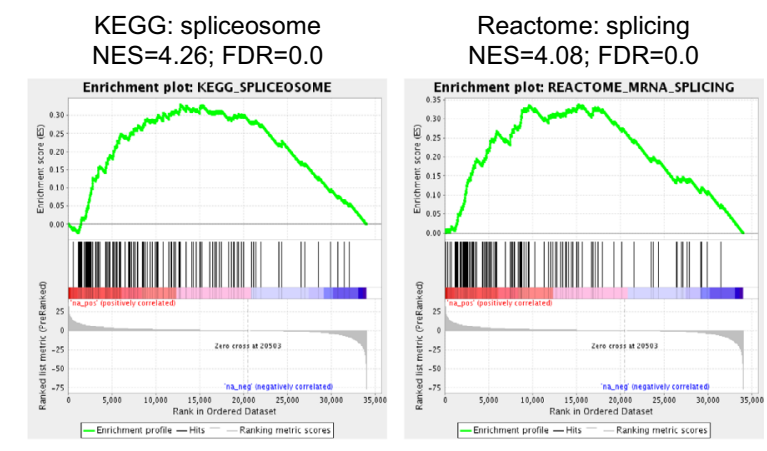

C

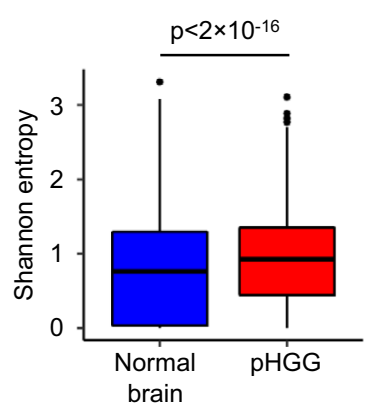

d

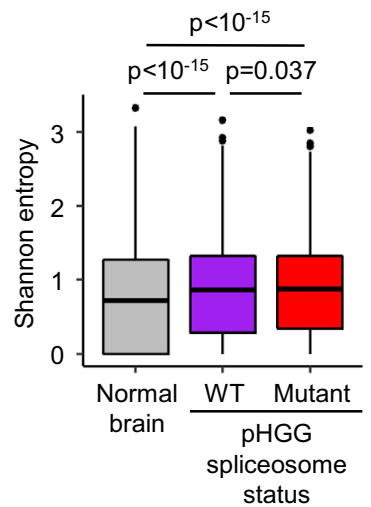

e

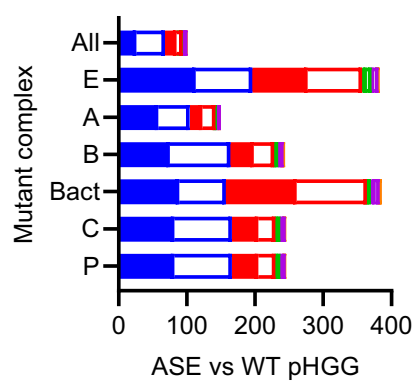

Fig. 1 The landscape of alternative splicing in pHGG. a Frequency of spliceosome mutations in pHGG in total (Spliceosome) and by subcomplex. b Gene set enrichment analysis (GSEA) comparing pHGG $(n=64)$ and normal brain $(n=20)$ for spliceosome components and mRNA splicing pathway. NES normalized enrichment score, FDR false discovery rate. c Splicing burden, as measured by the median Shannon entropy of multi-isoform genes, was plotted for normal brain $(n=20)$ and pHGG $(n=64)$. The box shows the interquartile range $(I Q R)$, the line shows the median, and the whiskers extend to $1.5 \times 1 Q R$. d Splicing burden was plotted for normal brain $(n=20)$ plus pHGG with wild-type (WT, $n=24)$ or mutant $(n=28)$ spliceosomes. The box shows the interquartile range (IQR), the line shows the median, and the whiskers extend to $1.5 \times \mid \mathrm{QR}$. e Differential splicing events (ASE; splice change $|\Delta \psi|>0.15$, FDR $<0.05$ ) were identified in pHGG with mutations in each spliceosome complex relative to pHGG with WT spliceosomes. Each bar shows the number of each type of event that was preferentially included (Inc) or skipped in mutant pHGG. SE skipped exon, MXE mutually exclusive exon, A5SS alternative $5^{\prime}$ splice site, A3SS alternative $3^{\prime}$ splice site, RI retained intron (see Supplementary Fig. S2a for schematic). Statistical tests: Wilcoxon rank-sum test (c), Pairwise Wilcoxon rank-sum tests with Benjamini-Hochberg correction (d). Source data are provided as a Source Data file.

spliceosome-mutant pHGG have an increased burden of ASEs, which varied with the component of the spliceosome complex that was mutant (Fig. 1e and Supplementary Fig. S1k).

Differentially spliced genes were enriched in the RAS/MAPK $\left(P<2 \times 10^{-4}\right)$, chromatin $\left(P=1.3 \times 10^{-4}\right)$, cellular communication $\left(P<3 \times 10^{-4}\right)$, and trafficking $\left(P=3 \times 10^{-9}\right)$ pathways (Fig. $2 \mathrm{~b}$ ), with very similar findings among the genes identified by SUPPA2 (Supplementary Fig. S2g). Remarkably, genes in the Catalogue of Somatic Mutations in Cancer (COSMIC) cancerdriver census ${ }^{20}$ were enriched significantly more than expected $\left(P=3 \times 10^{-26}\right)$, with 78 genes (8\%; 185 ASE) differentially spliced in $\mathrm{pHGG}$, in particular from the RAS/MAPK and chromatin pathways (Supplementary Fig. S3a, b). Furthermore, among oncogenic pathways curated by TCGA ${ }^{9}$, the RTK/RAS/ MAPK and PI3K pathways were significantly affected by splicing but not by expression (Supplementary Fig. S3c). The majority of differential ASE in cancer-driver genes were predicted to affect protein domains (53\%), intrinsically disordered regions $(50 \%)$ or PTMs (45\%; Fig. 2c, d).

Collectively, these data imply that pHGG frequently activates oncogenic processes non-mutationally. Accordingly, the rate of recurrent cancer-driver splicing was significantly higher than mutation in pHGG within both individual samples and genes (both $P<10^{-15}$; Fig. 2e). To test if the frequency of AS, and the convergence on cancer drivers, was a more general phenomenon, we used TCGA data for 18 tumor types with available normal tissue RNA-Seq. Remarkably, 17 had significantly higher cancerdriver splicing burden per sample than mutations $\left(P \leq 10^{-17}\right)$, with most also having more recurrent AS alterations than mutations (Fig. 2f and Supplementary Fig. S3d, e).

Adult diffuse gliomas (glioblastoma, GBM; lower-grade glioma; LGG) had the highest level of splice-driven alterations (Fig. 2f, g). Those with the highest differential cancer-driver splicing had significantly worse overall survival (OS) $\left(P<10^{-15}\right)$ while those with higher cancer-driver mutation burdens did not (Supplementary Fig. S4a, b). Adult diffuse glioma occurs in three main subgroups defined by isocitrate dehydrogenase (IDH) mutations and co-deletion of chromosomes $1 p$ and $19 q^{4}$. IDH wild-type (IDH-wt) GBM have poor outcome compared with IDH-mutant diffuse glioma (with (IDH-mut/codel) or without (IDH-mut/non-codel) $1 \mathrm{p} / 19 \mathrm{q}$ codeletion) ${ }^{4}$, and GBM had increased cancer-driver AS (Supplementary Fig. S4c). However, on multivariate survival analysis the effect of cancer-driver AS on OS was subgroup-independent $(P=0.01$; Supplementary Fig. S4d). Splicing, but not mutation burden, was significantly associated with worse OS for IDH-wt GBM $(P=0.007)$ and IDH-mut/non-codel astrocytoma $(P=0.015)$, with a trend $(P=0.18)$ for IDH-mut/codel oligodendroglioma (Fig. $2 \mathrm{~h}$, i and Supplementary Fig. S4e-i).

To test the dependence of HGG on the spliceosome, we treated both pHGG, GBM, and normal human astrocyte cell lines with 
a

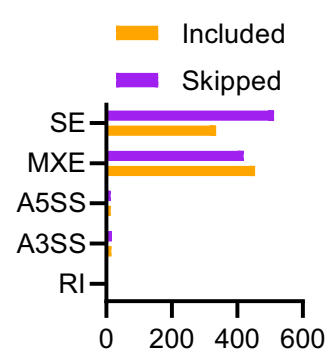

Differential events

$(\mathrm{D} \psi>0.15, \mathrm{FDR}<0.05)$ b

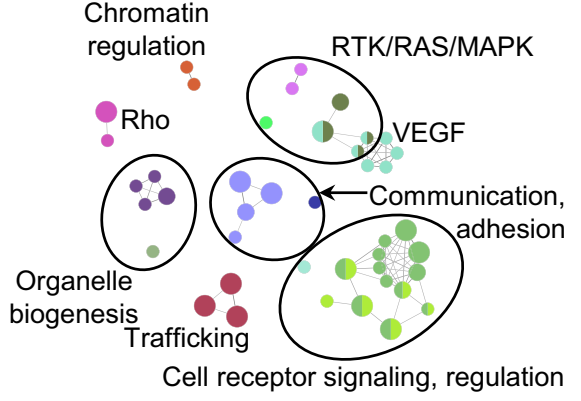

f
C

Domain-affecting ASE

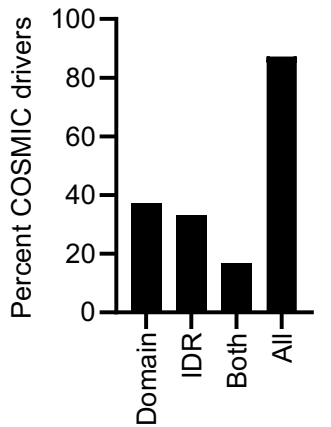

g d

PTM-affecting ASE
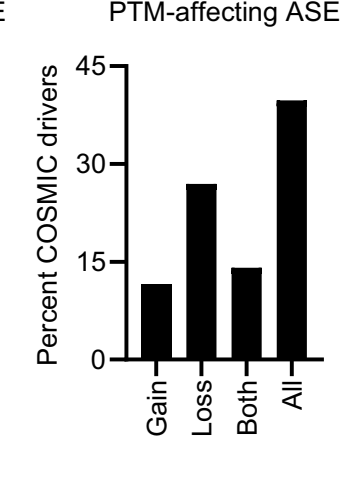

e

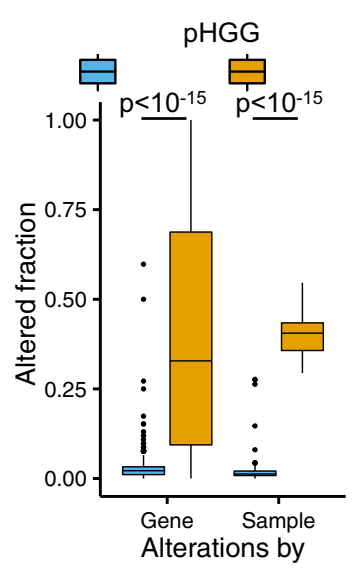

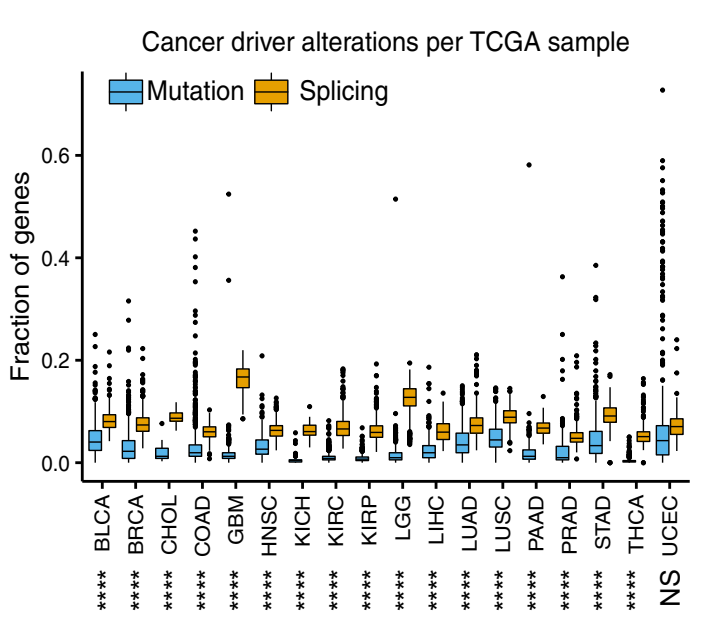

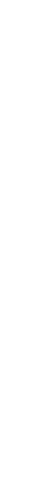


the spliceosome inhibitor pladienolide B. All lines including normal human astrocyte controls were exquisitely sensitive to this drug (IC50 0.76-4.30 nM; Fig. 2j). Similar results were obtained with a second inhibitor, madrasin (Supplementary Fig. S4j), demonstrating that, although unlikely to be directly targetable, HGG cells have a fundamental requirement for spliceosome activity to remain viable.

Functional consequences of chromatin regulator disruption by alternative splicing. While the frequency of AS in cancer genes and its association with more aggressive tumor behavior are intriguing, an important question is the potential for functional effects of these splicing events. We therefore looked more closely at the functional implications of the chromatin modifier and RAS/MAPK pathway ASEs we found in HGG.

Chromatin disruption through recurrent H3K27M mutations and DNA hypomethylation have been characterized in $\mathrm{pHGG}^{1,2,21}$. Our data suggest AS may also affect epigenetic regulation of $\mathrm{pHGG}$, with chromatin modifiers more affected by splicing than expression changes (Figs. $2 \mathrm{~b}$ and $3 \mathrm{a}$ ). Strikingly, among the 48 differentially spliced chromatin regulators, several protein complexes had multiple affected members, in particular mammalian switch/sucrose-nonfermentable (SWI/SNF), nucleosome remodeling and deacetylase ( $\mathrm{NuRD})$, and polycombrepressive complex 1.1 (PRC1.1; Fig. 3b, c). We validated two, MBD1 and SMARCC1, by RT-PCR (Supplementary Fig. S2d). Interestingly, the SWI/SNF complex has previously been implicated in alternative splicing of its target genes ${ }^{22}$, which were also enriched in pHGG differential ASE, with high proportions correlating significantly with the splicing of individual complex members (Fig. 3d, e). These data implicate AS in epigenetic regulation at the level of chromatin regulators and their downstream target genes.

To further investigate this, we analyzed the predicted effects of differential ASE in chromatin regulators. Most (95\%) of the AS involved protein domains (49\%), intrinsically disordered regions (67\%) and/or PTMs (44\%), suggesting that AS in chromatin regulators will affect their function (Fig. 3f, g).

Activation of RAS/MAPK signaling by an NF1 isoform switch in HGG. RTK/RAS/MAPK pathway ASEs were also strikingly enriched in HGG. FGFR1, NTRK3, NF1, and BRAF all had highly ranked ASEs involving key structural features which would be predicted to affect RAS/MAPK activation (Fig. 4a-d and Supplementary Fig. S5). We validated the FGFR1 and NF1 ASEs in RNA-Seq from a small independent pHGG cohort ( $\mathrm{pHGG}$ $(n=9)$, normal brain $(n=3)$; Supplementary Fig. S5g, h $)^{23}$ as well as by RT-PCR (Supplementary Fig. S2d).

$B R A F$ splicing in the RAS-binding domain (Supplementary Fig. S5a, b) was predicted to remove BRAF-S151, which is subject to feedback through phosphorylation by ERK to limit RAS/ MAPK activation ${ }^{24}$. NTRK3 splicing is predicted to remove the Y516 autophosphorylation site that is important for RAS activation (Supplementary Fig. S5c, d) ${ }^{25}$. Thus, BRAF and NTRK3 splicing would be expected to increase and decrease MAPK activation, respectively; however, their differential expression in pHGG would oppose this, making interpretation of their functional relevance more challenging (Supplementary Fig. S5b, d). FGFR1 is expressed in two isoforms. FGFR1- $\beta$ skips the $\mathrm{N}$-terminal Ig-like domain, has a higher affinity for FGF than FGFR1- $\alpha$ leading to increased downstream signaling and can be skipped in $\mathrm{GBM}^{26,27}$. It was also skipped in pHGG, accompanied by FGFR1 upregulation (Supplementary Fig. S5e, f).

NF1 exon23a was among the most significant ASE in pHGG (mean $\psi$ increase $46 \%, P<10^{-15}$; Fig. $4 \mathrm{~b}-\mathrm{d}$ ). This exon is skipped in NF1-I, while its inclusion in NF1-II inserts a 21-aa loop into the RAS-GTP-binding site, reducing the affinity of NF1 for RASGTP28,29. NF1-II is the predominant non-brain isoform, while NF1-I is expressed in the brain $\left(P<10^{-15}\right.$; Supplementary Fig. S5i). Importantly, bulk expression of NF1 is unchanged in pHGG and does not correlate with exon23a inclusion (Supplementary Fig. S5j, k). Finally, we further confirmed the differential splicing of NF1 exon23a using qRT-PCR in three matched pHGG-normal pairs using isoform-specific primers (Supplementary Fig. S51). Interestingly, we also observed the same isoform switch in three mouse pHGG models ${ }^{23,30,31}$ indicating this is a conserved feature between species (Supplementary Fig. S5m).

The NF1-II isoform switch was also evident in adult GBM and IDH-mutant diffuse gliomas (mean $\psi$ increase $50-62 \% ; P=0.01$ [junction], $P \leq 0.05$ [isoform] Fig. 4e and Supplementary Fig. S6a). The isoform switch did not correlate strongly with NF1 expression, although NF1 was modestly down- and upregulated in IDH-wt and IDH-mut/codel diffuse glioma, respectively (Supplementary Fig. S6b-e).

NF1-II has been reported to decrease RAS-GTP turnover, boosting downstream MAPK activity ${ }^{32,33}$. To test the functional consequence of this switch in PHGG and GBM, we blocked NF1 exon23a inclusion with morpholinos targeting the $3^{\prime}$ and $5^{\prime}$ intron-exon junctions to interfere with spliceosome access. These NF1 exon23a-specific morpholinos promoted exon23a skipping and increased MAPK signaling in both pediatric and adult HGG cells (Fig. 5a and Supplementary Fig. S7a). Accordingly, increased NF1 exon23a-high adult diffuse glioma patients (Q4) have significantly increased phospho-ERK $(P \leq 0.02$; Fig. 5b). Furthermore, gene sets transcriptionally regulated by RAS or MAPK, which are overall highly upregulated in both pHGG and adult diffuse glioma (Fig. S7b-d), are significantly upregulated in tumors with increased NF1 exon23a inclusion in a manner not related to bulk NF1 expression (Fig. 5c, d and Supplementary Fig. S7e-g). In contrast, there was no increased RAS/MAPK activation associated with RAS/MAPK mutant pHGG or adult diffuse glioma (Fig. 5e, f, Supplementary Fig. S7h, and Supplementary Data 2).

The functional RAS/MAPK activation by NF1 isoform switching prompted us to ask whether it was associated with OS. After excluding NF1-mutant patients, increased NF1 exon23a inclusion was associated with worse OS in adult diffuse glioma $\left(P=2 \times 10^{-5}\right.$; Supplementary Fig. S7i). This was specific to IDH-wt GBM $(P=0.028)$ and was independent of NF1 expression and RAS/MAPK pathway gene mutation on multivariate analysis $(P=0.039)$ (Fig. $5 \mathrm{~g}, \mathrm{~h}$ and Supplementary Fig. S7j, k).

Recent work has shown that NF1 exon23a splicing is regulated by neuronal differentiation and that altering the inclusion rate affects differentiation kinetics ${ }^{34}$, raising the prospect that NF1 exon23a splicing may also play a role in the differentiation state of HGG. We used an established model of neuronal differentiation of $\mathrm{GBM}^{35}$, in which culturing cells in the presence of sodium butyrate in the absence of serum induces differentiation (Supplementary Fig. S8a). We transfected cells with control or NF1-specific morpholinos for $24 \mathrm{~h}$ to establish a specific splicing pattern, followed by $48 \mathrm{~h}$ of differentiation. In this system, U87 GBM cells exhibited robust induction of differentiation markers compared to untreated cells (Supplementary Fig. S8b). In contrast to neuronal differentiation of PC12 cells where NF1 exon23a inclusion decreases ${ }^{34}, N F 1$ exon23a inclusion did not change in differentiated U87 cells (Supplementary Fig. S8c).

In differentiating PC12 cells, modulating NF1 exon23a inclusion affected the dynamics of neuronal differentiation ${ }^{34}$. U87 cells transfected with NF1-specific morpholinos to block the inclusion of exon23a did not change the expression of neuronal 


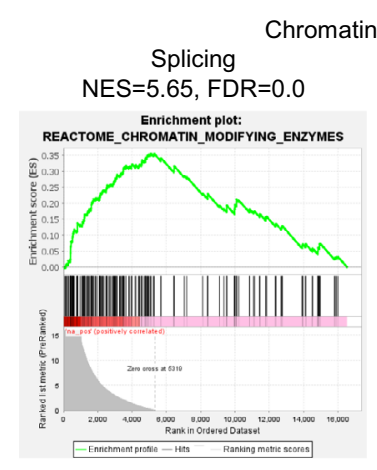

C

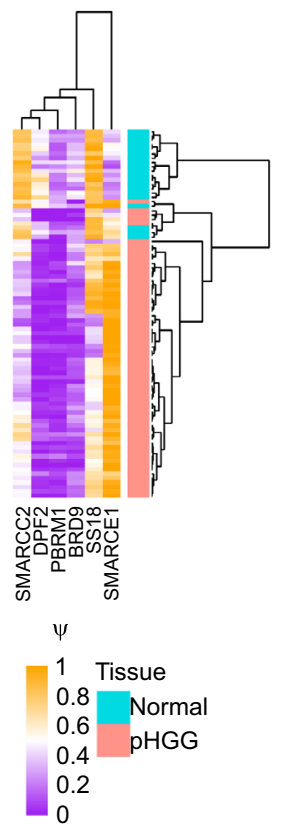

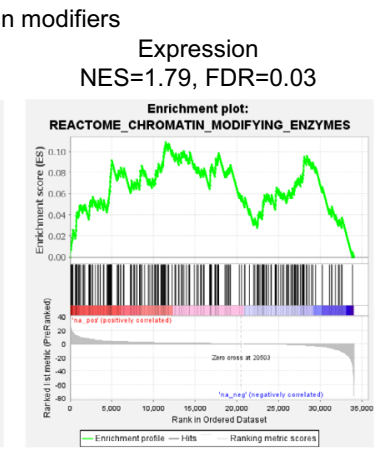

d

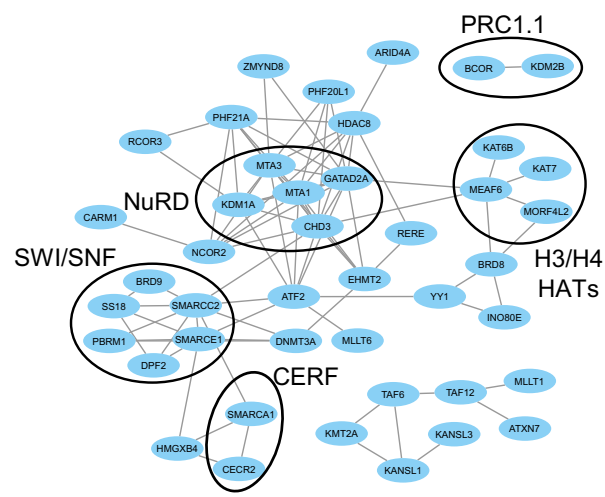

e

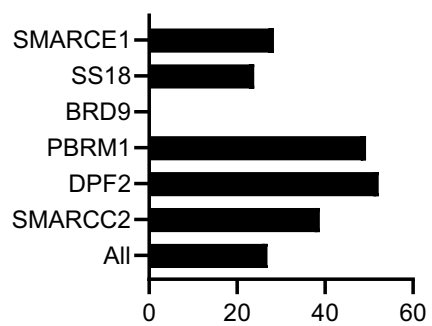

Percent SWI/SNF target gene splicing correlating with subunit splicing

g
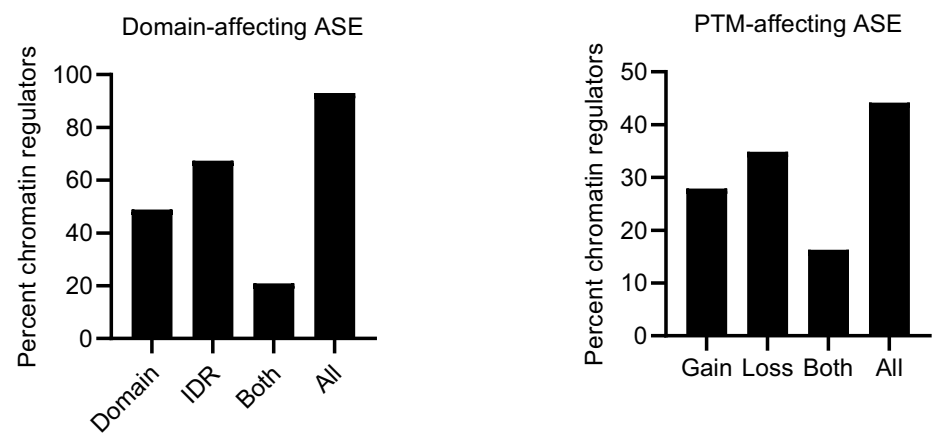

Fig. 3 Chromatin regulators are disrupted by alternative splicing in pHGG. a Gene set enrichment analysis of chromatin-modifying enzymes among genes differentially spliced (left) or differentially expressed (right) in pHGG. NES normalized enrichment score, FDR false discovery rate. b Protein-protein interactions between chromatin-related proteins that are differentially spliced in pHGG were retrieved from STRING. Edge thickness represents interaction confidence. SWI/SNF mammalian switch/sucrose-nonfermentable, NuRD nucleosome remodeling and deacetylase, PRC1.1 polycomb-repressive complex 1.1, CERF CECR2-containing remodeling factor complex. c Heatmap of percent inclusion ( $\psi$ ) of differential splicing events $(|\Delta \psi|>0.15, \mathrm{FDR}<0.05)$ identified between pHGG $(n=64)$ and normal brain $(n=20)$ in SWI/SNF complex members. d Gene set enrichment analysis (GSEA) of SWI/SNF target gene enrichment among genes differentially spliced in pHGG. NES normalized enrichment score, FDR false discovery rate. e Proportion of SWI/SNF target genes whose $\psi$ correlates significantly (Benjamini-Hochberg corrected $P<0.05$ ) with $\psi$ of each SWI/SNF complex member across pHGG samples $(n=64)$, or with the maximally variant complex member ("All") in each sample. $\mathbf{f}$ Percentage of chromatin regulators with differential ASE predicted to affect structural domains and intrinsically disordered domains (IDR) mapped in UniProt. $\mathbf{g}$ Percentage of chromatin regulators with differential ASE predicted to affect post-translational modification (PTM) sites mapped in UniProt. Statistical tests: $t$ test with Benjamini-Hochberg multiple hypothesis testing correction (e). Source data are provided as a Source Data file.

marker genes in either media condition compared to cells transfected with control morpholino, suggesting that NF1 exon23a inclusion does not affect HGG cell differentiation in this system and conditions (Supplementary Fig. S8b).

Collectively, these data show that oncogenic pathways can be functionally activated through non-mutagenic processes, such as
AS, in HGG, and suggest that mutation profiling alone may be insufficient to identify patients for targeted therapies.

Alternative splicing of NF1 exon23a in pHGG is regulated downstream from REST. As a family, splicing factors are widely differentially expressed in pHGG (Fig. 1b). We compared the 
a

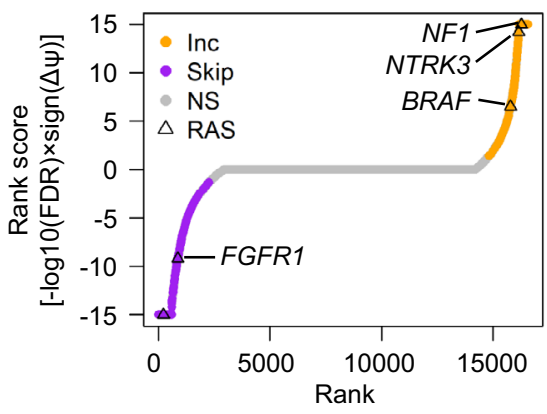

C

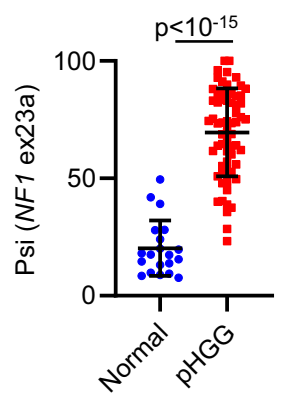

b
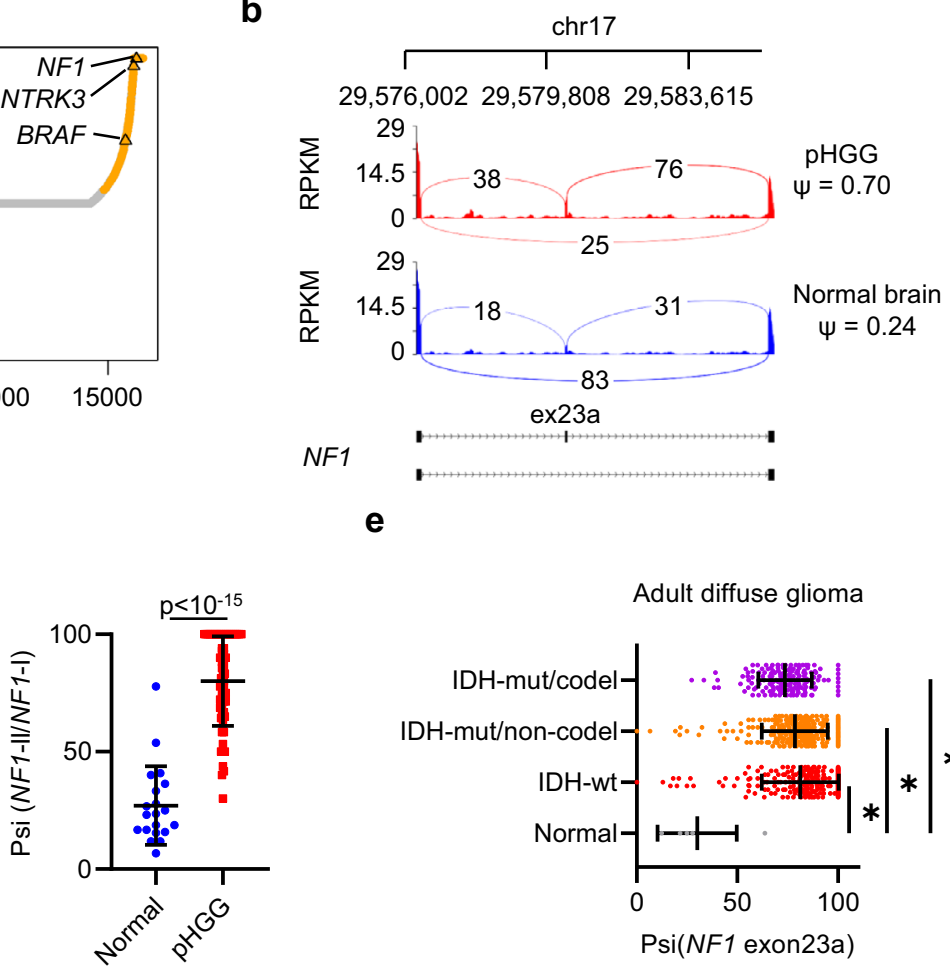

Adult diffuse glioma

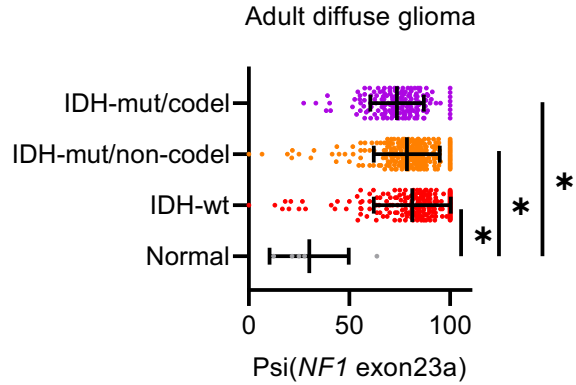

Fig. 4 Alternative splicing of NF1 exon23a in HGG. a Genes were ranked according to their most significant splicing score. Significance is shown by point color (orange, inclusion [Inc]; purple, skipping; NS not significant). RAS/MAPK pathway genes with significant differential ASE (ASE; $|\Delta \psi|>0.15$, FDR < 0.05) are marked. $\mathbf{b}$ Sashimi plot showing average reads per kilobase per million (RPKM) in pHGG and normal brain for coverage of the NF1 exon23a skipped exon and immediate up- and downstream exons. c NF1 exon23a percent inclusion (psi) in pHGG $(n=64)$ and normal brain $(n=20)$ as determined by junction coverage. Bars show mean \pm standard deviation. d NF1 exon23a inclusion in pHGG and normal brain was determined from the ratio of isoform expression of NF1-I and NF1-II transcripts in PHGG $(n=64)$ and normal brain $(n=20)$ as determined by junction coverage. Bars show mean \pm standard deviation. e NF1 exon23a percent inclusion (psi) based on NF1 transcript ratios in TCGA diffuse glioma dataset: adult normal brain ( $n=5$ ) and IDH-wt ( $n=231 ; P=0.01)$, IDH-mut/non-codel $(n=256 ; P=0.01)$ and IDH-mut/codel $(n=170 ; P=0.02) \mathrm{HGG}$. Bars show mean \pm standard deviation.

Statistical tests: $t(\mathbf{c}, \mathbf{d})$, Brown-Forsythe ANOVA with Dunnett's T3 multiple comparisons test $(\mathbf{e}) .{ }^{\star} P<0.05$. Source data are provided as a Source Data file.

differential expression of RNA-binding proteins including splice factors with enrichment of their binding motifs in the introns upand downstream from differentially spliced exons, finding that members of the CELF and ELAVL families are the most downregulated genes with enriched motifs (Fig. 6a). IGF2BP2/3, which is known to promote tumorigenic behavior through stabilization of HMGA mRNA and is widely overexpressed in cancer ${ }^{36}$, is strongly upregulated in $\mathrm{pHGG}$ with accompanying enrichment of its binding motif (Fig. 6a).

The CELF/ELAVL enrichment was intriguing given that the CELF/ELAVL families have been reported to repress NF1 exon23a inclusion while the MBNL and TIA1/TIAL1 families promote it ${ }^{37,38}$ (Supplementary Fig. S9a). Consistent with intronic CELF/ELAVL binding sites surrounding exon23a, their motifs are enriched both up- and downstream of differentially spliced exons in pHGG, respectively (Fig. $6 \mathrm{~b}$ and Supplementary Fig. S9a). Furthermore, the CELF/ELAVL families, in particular CELF3-5 and ELAVL2-4, are downregulated in pHGG while TIA/ TIAL1 and MBNL3 are modestly upregulated (Fig. 6c and Supplementary Fig. S9b). The same pattern was found in adult diffuse glioma, albeit less pronounced in IDH-mut/codel oligodendroglioma (Supplementary Fig. S9c-e).

We hypothesized that family-wide CELF/ELAVL downregulation could explain the increased NF1 exon23a inclusion we observed in HGG. By expressing CELF4 and ELAVL3 in U343 GBM and HEK293T cells, we confirmed by RT-PCR that they suppress inclusion of NF1 exon23a (Fig. 6d and Supplementary
Fig. S6f). The enrichment of CELF/ELAVL motifs surrounding differentially spliced exons imply they regulate many more differential ASE in pHGG. To assess this possibility, we identified genes with binding sites surrounding differential exons. By RTPCR we could validate select additional targets beyond NF1, including the FGFR1 ASE (Fig. 6d). Importantly, for each event we tested, the expression of CELF4/ELAVL3 modulated its splicing in the opposite direction to that induced in $\mathrm{pHGG}$, supporting the idea that these families regulate the pHGG AS landscape.

Increased inclusion of NF1 exon23a in HGG leads to increased RAS/MAPK activation (Fig. 5 and Supplementary Fig. S7). We therefore next compared the expression of CELF/ELAVL genes in pHGG with RAS activity as well as NF1 exon23a inclusion, finding that these families (especially CELF3-6 and ELAVL2-4) are significantly negatively correlated with both NF1 exon23a inclusion and RAS activity (Fig. 6e).

In all, $14 \%(n=13 / 90)$ of pHGG had an SNV $(n=9)$ or CNV $(n=4)$ in a CELF/ELAVL family gene, suggesting that both mutagenic and non-mutagenic processes are responsible for this mechanism. To identify potential regulators of these genes, we profiled ENCODE ChIP-Seq data to find transcription factors binding their promoters. The significant hits across all cell lines were near-exclusively the RE1 silencing transcription factor (REST; Fig. 7a), which has recently been shown to be important for pHGG and GBM cell growth amongst other cancers ${ }^{39-42}$. Strong REST peaks ${ }^{43}$ were observed in GBM cells associated with 
a

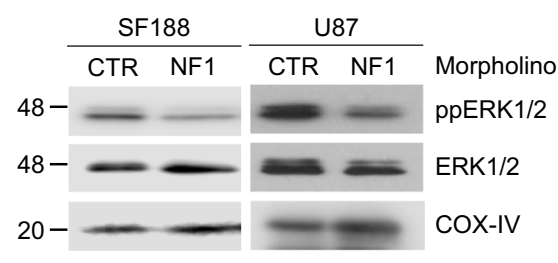

b

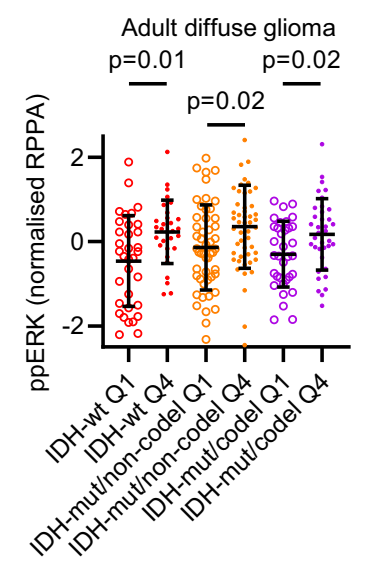

C

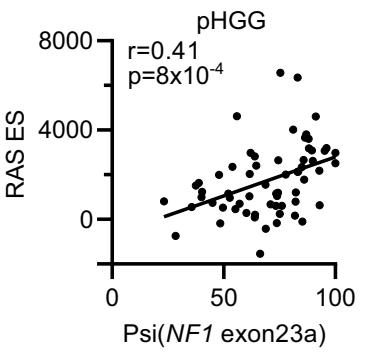

d

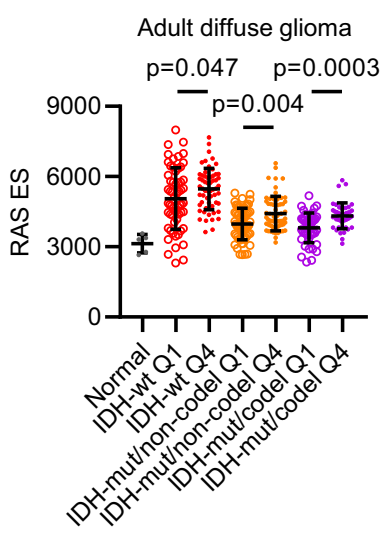

g

IDH-wt adult GBM

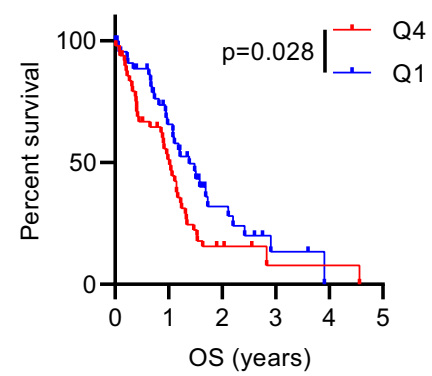

e

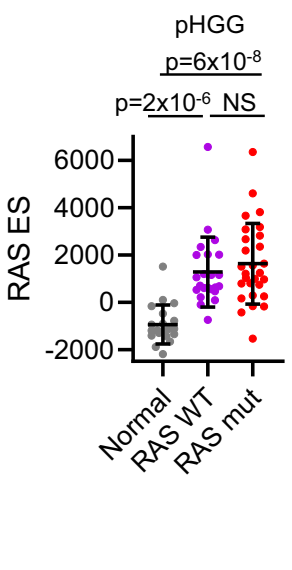

f

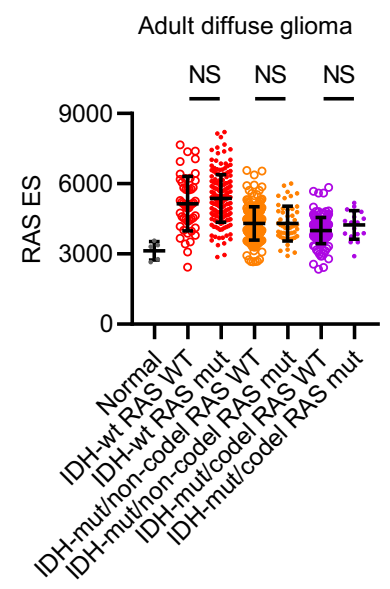

h

\begin{tabular}{c|ccc|ccc}
\multirow{2}{*}{ IDH-wt adult GBM } & \multicolumn{3}{|c|}{ Univariate } & \multicolumn{3}{c}{ Multivariate } \\
\cline { 2 - 7 } & $\mathrm{HR}$ & $95 \% \mathrm{Cl}$ & $\mathrm{p}$-value & $\mathrm{HR}$ & $95 \% \mathrm{Cl}$ & $\mathrm{p}$-value \\
\hline Exon23a Q4 v Q1 & 1.78 & $1.11-2.77$ & 0.010 & 1.81 & $1.06-3.09$ & 0.039 \\
RAS Mut v WT & 1.07 & $0.73-1.58$ & 0.72 & 0.92 & $0.50-1.69$ & 0.78 \\
NF1 high v low & 0.65 & $0.40-1.063$ & 0.086 & 0.70 & $0.41-1.18$ & 0.18
\end{tabular}

Fig. 5 Activation of RAS/MAPK by NF1 exon23a inclusion in HGG. a Western blot of cells harvested 48 h post transfection with control or NF1 exon23aspecific morpholinos. Gels are representative of two experiments. b Normalised reverse-phase protein array (RPPA) signal for phospho-ERK (ppERK) for adult diffuse glioma Q1 and Q4 patients in each subgroup. n: IDH-wt Q1=34, Q4=30; IDH-mut/non-codel Q1=50, Q4=51; IDH-mut/codel Q1 = 34, $\mathrm{Q} 4=37$. Bars show mean \pm standard deviation. c Plot of NF1 exon23a inclusion (psi) and RAS pathway activation score (RAS ES; enrichment score, determined from single-sample GSEA [ssGSEA]) in pHGG. r: Pearson correlation. d RAS pathway activation ssGSEA score for the normal brain ( $n=5$ ) and adult diffuse glioma patients that are in quartile 1 (Q1) and quartile 4 (Q4) of NF1 exon23a inclusion for each subgroup. n: IDH-wt Q1=60, Q4 = 58; IDHmut/non-codel $\mathrm{Q} 1=63, \mathrm{Q} 4=63 ; \mathrm{IDH}$-mut/codel Q1 =45, Q4=43. Bars show mean \pm standard deviation. e RAS pathway activation ssGSEA score in normal brain $(n=20)$ or pHGG that is RAS/MAPK WT $(n=23)$ or mutant $(n=28)$. Bars show mean \pm standard deviation. $\mathbf{f}$ RAS pathway activation sSGSEA score for adult diffuse glioma subgroups that are RAS/MAPK WT or mutant. n: IDH-wt WT $=27$, mut $=216 ;$ IDH-mut/non-codel WT $=165$, mut $=105 ; \mathrm{IDH}$-mut/codel WT $=165$, mut $=105$. Bars show mean \pm standard deviation. $\mathbf{g}$ Kaplan-Meier survival plot of (NF1-WT) IDH-wt GBM patients that are in the top (Q4, $n=50$ ) or bottom (Q1, $n=47$ ) quartile of NF1 exon23a inclusion. $\mathbf{h}$ Univariate and multivariate Cox proportional-hazards survival analysis of adult IDH-wt GBM accounting for NF1 exon23a inclusion (Q4 vs Q1), RAS/MAPK pathway mutation, and NF1 expression. HR hazard ratio, Cl confidence interval. Statistical tests: $(\mathbf{b}, \mathbf{c}, \mathbf{d}, \mathbf{f})$, Brown-Forsythe ANOVA with Dunnett's T3 multiple comparisons test (e), Gehan-Breslow-Wilcoxon (g), Cox proportional-hazards (h). NS not significant. Source data are provided as a Source Data file. 
a

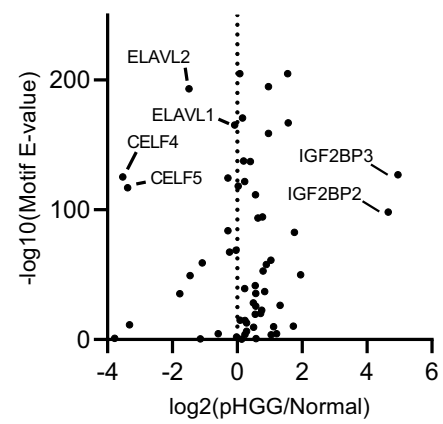

C

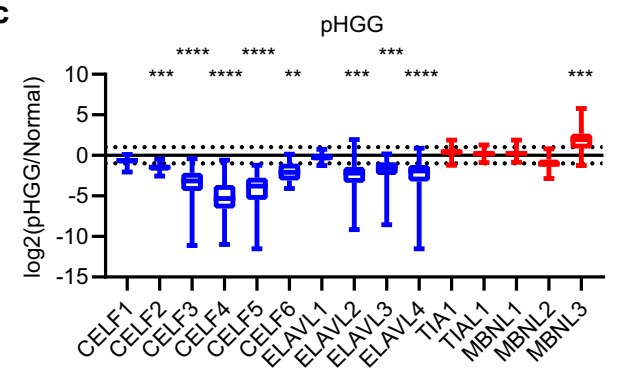

e

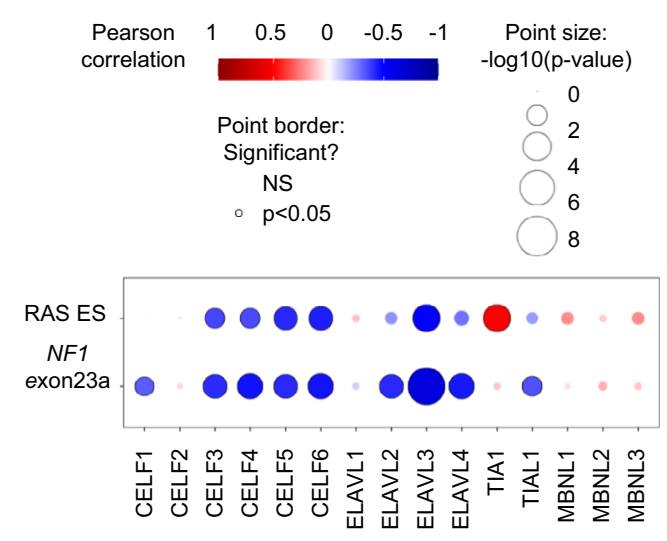

b

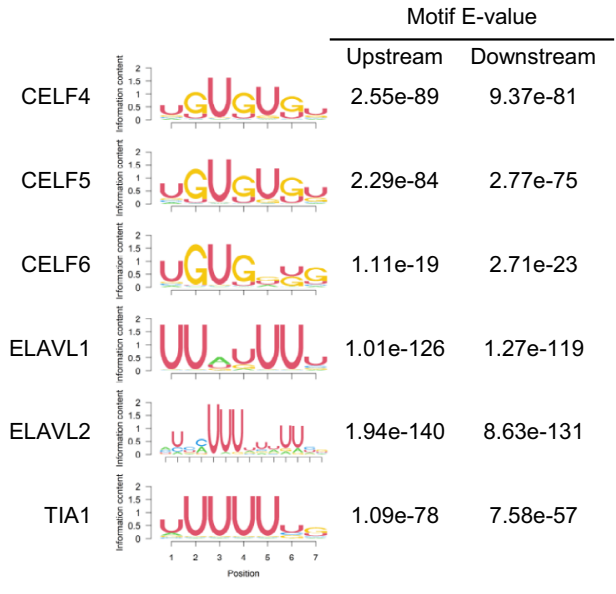

d

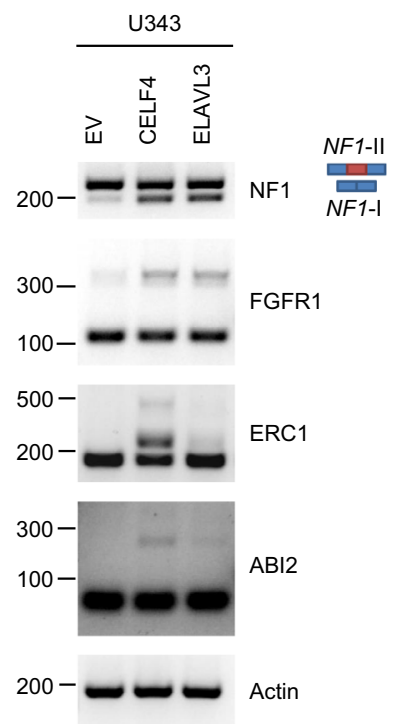

Fig. 6 NF1 exon23a splicing in HGG is controlled by differential expression of the CELF/ELAVL gene families. a Scatter plot comparing log2 fold change in expression between pHGG and normal brain of splice factors and RNA-binding proteins, with the enrichment of the RNA motifs they bind in introns surrounding differentially spliced exons in pHGG. $\mathbf{b}$ Motifs and enrichment in up- and downstream introns surrounding differentially spliced exons for the indicated splicing factors. c Expression ratio (log2 fold change) of negative (blue) and positive (red) NF1 exon23a splicing regulators between pHGG $(n=64)$ and normal brain $(n=20)$. Box shows IQR with the median marked, and the whiskers show the range. $P$ values: CELF2, 0.0001; CELF3, $4 \times 10^{-16}$; CELF4, $7 \times 10^{-28}$; CELF5, $2 \times 10^{-26}$; CELF6, 0.001; ELAVL2, 0.0003; ELAVL3, 0.0001; ELAVL4, $1.5 \times 10^{-5} ;$ MBNL2, 0.01, MBNL3, $7 \times 10^{-5}$. d U343 GBM cells transduced with control (EV) lentivirus or expressing CELF4 or ELAVL3 were analyzed by RT-PCR for the indicated splicing events. Bands corresponding to NFT-II (includes exon23a, red box) and NFT-I are shown at the left. Gels are representative of two experiments. e Bubble plot showing the relationship between NF1 exon23a splicing regulators and NF1 exon23a inclusion and RAS activity score (ES, enrichment score from ssGSEA) in pHGG. Bubble color represents Pearson correlation, size is significant, and statistically significant correlations have a black border on the bubble. Statistical tests: $t(\mathbf{c}, \mathbf{e}) .{ }^{\star} P<0.05,{ }^{\star \star} P<0.01,{ }^{\star \star \star} P<0.001$. Source data are provided as a Source Data file.

NF1 exon23a splice regulators, which we confirmed for the same sites in CELF3, CELF4, and ELAVL3 in three primary pHGG cell lines by ChIP-qPCR (Fig. 7b and Supplementary Fig. S9g).

REST was significantly upregulated in pHGG $(P<0.0001)$ and, as expected for a repressive transcription factor, expression of RE1 motif-containing, REST-bound genes was highly downregulated (Fig. $7 \mathrm{c}, \mathrm{d})^{44}$. In particular, REST directly binds most CELF/ELAV and is negatively associated with their repression (Fig. 7e), suggesting that it binds and represses their expression in pHGG. To test this hypothesis, we depleted REST from primary pHGG cells, finding significant increases in CELF3, CELF4, and ELAVL3 expression (Fig. 7f and Supplementary Fig. S9h). In turn, REST depletion caused a significant increase in NF1-I expression with an accompanying decrease in NF1-II (Fig. 7g). Consistent with this, increased REST 
a

ENCODE ChIP-Seq enrichment

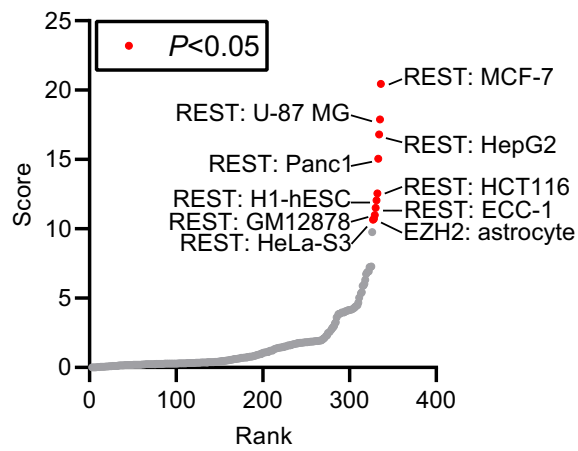

d

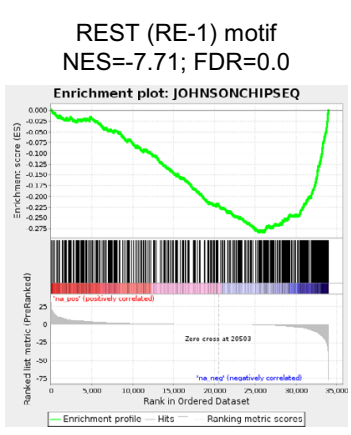

e

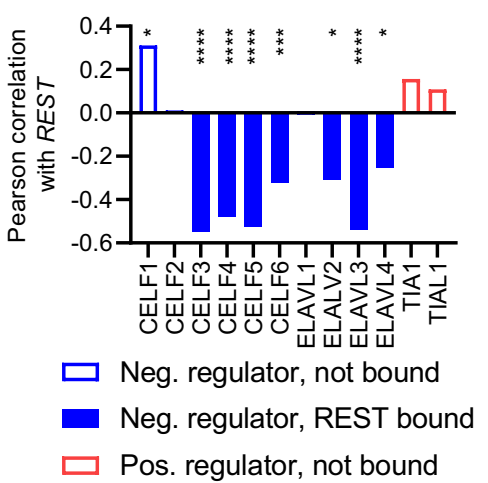

g

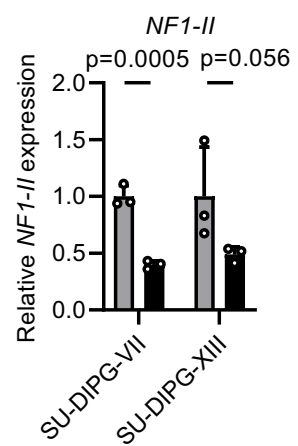

h

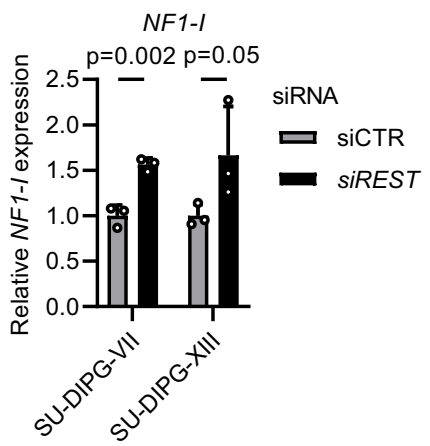

b

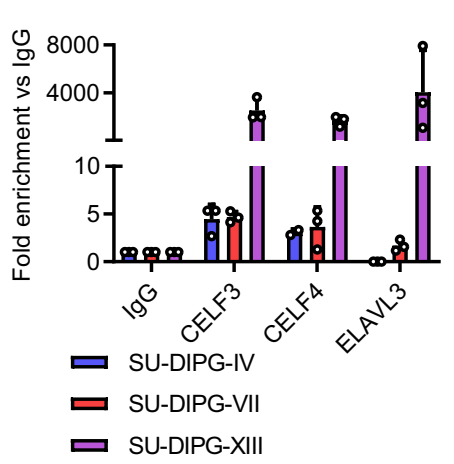

C

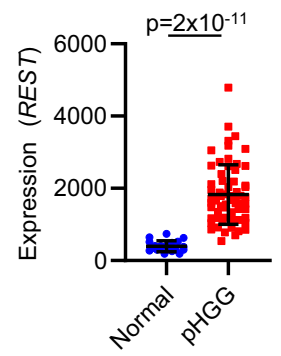

f

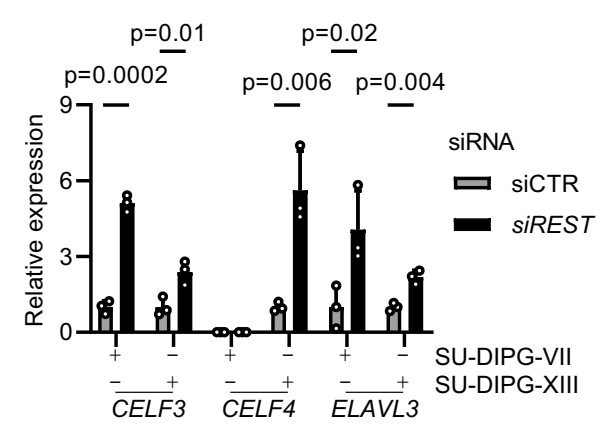

i

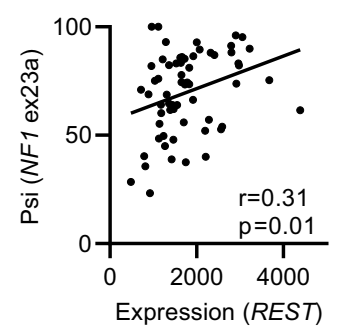

PHGG

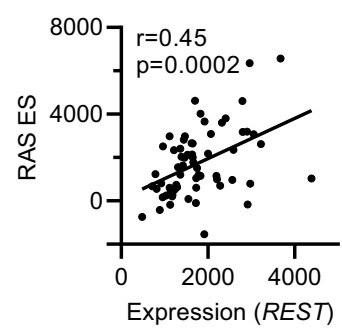

Fig. 7 NF1 exon23a splicing in pHGG is regulated downstream from REST. a Enrichment of transcription factor binding sites in NF1 exon23a splice regulators were calculated by Enrichr using ENCODE ChIP-Seq data. All significant results (FDR $<0.05$, red) are highlighted red and the dataset labeled. b ChIP was carried out using REST or IgG antibodies with chromatin from primary DIPG cell lines and analyzed as percent input at the indicated loci $(n=3)$. Bars show mean \pm standard deviation. c REST expression in pHGG $(n=64)$ and normal brain $(n=20)$. Bars show mean \pm standard deviation. $\mathbf{d}$ Gene set enrichment analysis of REST signature genes with a REST-bound RE1 motif. NES normalized enrichment score, FDR false discovery rate. e Pearson correlation of REST expression and expression of NF1 exon23a splice regulators (CELF1, $P=0.01 ; \mathrm{CELF3} P=10^{-5}, \mathrm{CELF} 4, P=10^{-4}, \mathrm{CELF5}, P=10^{-6}$, CELF6, $P=0.01$; ELAVL2, $P=0.01$; ELAVL3, $P=10^{-5}$, ELAVL4, $P=0.04$ ). Genes bound by REST across ENCODE ChIP-Seq datasets are shaded in solid color, genes not bound by REST are empty. $\mathbf{f}$ qRT-PCR in SU-DIPG-VII and SU-DIPG-XIII cell lines transfected with control or REST-specific siRNA. Bars show mean \pm standard deviation $(n=3)$ relative to $18 \mathrm{~S}$ housekeeping, normalized to siCTR expression for each gene. $\mathbf{g}$ qRT-PCR in SU-DIPG-VII and SUDIPG-XIII cell lines transfected with control or REST-specific siRNA. Bars show mean \pm standard deviation $(n=3)$ relative to $18 \mathrm{~S}$ housekeeping, normalized to siCTR expression for each gene. $\mathbf{h}$ Scatter plot of REST expression and NF1 exon23a inclusion in pHGG. r: Pearson correlation. $\mathbf{i}$ Scatter plot of REST expression and RAS pathway activity (ssGSEA ES) in pHGG. r: Pearson correlation. Statistical tests: $t$ (all; multiple $t$ tests with two-stage linear step-up procedure of Benjamini, Krieger, and Yekutieli $(\mathbf{f}, \mathbf{g})$ ). ${ }^{\star} P<0.05,{ }^{\star \star} P<0.01,{ }^{\star \star \star} P<0.001,{ }^{\star \star \star}{ }^{\star \star} P<0.0001$. Source data are provided as a Source Data file.

expression was significantly associated with NF1 exon23a splicing and RAS/MAPK activation $(P \leq 0.01$; Fig. $7 \mathrm{~h}, \mathrm{i}$ and Supplementary Fig. S9i). Together, this supports a mechanism by which REST upregulation in pHGG drives increased RAS/MAPK signaling by promoting NF1 exon23a inclusion (Supplementary Fig. S9j).

\section{Discussion}

Here, we found that pHGG has increased alternative splicing (AS) burden compared with the normal brain. In some tumors, this is associated with spliceosome mutations. Previous work identified sporadic AS alterations in adult cancers, as well as mutations or 
expression changes in splicing factors and direct mutations to splice sites within genes ${ }^{10,45,46}$. We found the earliest assembly (complex E) and assembled catalytic (Bact) stages of the spliceosome to be particularly affected, suggesting that target site recognition and initiation of catalysis are the key determinants driving this pattern. Given the lethality of spliceosome inhibitors towards pHGG/GBM lines it is likely that the spliceosome mutations acquired in pHGG do not render the spliceosome completely inactive but rather alter the activity of the spliceosome. In addition to mutations in the spliceosome itself affecting AS in pHGG, not every case had mutations in the spliceosome suggesting other mechanisms are also at play, including differential expression of spliceosome components/splicing factors that could be regulated by transcriptional or epigenetic rewiring. Furthermore, the widespread epigenome remodeling that takes place in HGG suggests that this could also have an impact on AS given the known effects of histone and DNA methylation on splicing 47

Tumors each acquire mutations in a small subset of cancer drivers $3,9,20$. We found their burden of AS changes to be significantly higher, not only in pHGG but also in a broad range of adult cancers, implying that AS is an under-appreciated mechanism of oncogenic pathway activation. In adult diffuse glioma, which exhibited the biggest differences between cancerdriver mutation and AS burden, the latter also conferred a worse prognosis.

Differential ASEs in pHGG converged on cancer-driver genes, particularly the RAS/MAPK and chromatin modification pathways, and were predicted to have functional consequences. We showed that the NF1 isoform switch, which has previously been identified in RT-PCR analyses of adult $\mathrm{GBM}^{48,49}$, is driven by REST-mediated suppression of exon23a splice regulators and leads to activation of the RAS/MAPK pathway. Significantly, the NF1 isoform switch was more closely associated with signatures of RAS/MAPK activation than mutations in RAS/MAPK pathway member genes. Additional ASE such as that found in FGFR1 would also be expected to lead to elevated RAS/MAPK signaling.

Germline alterations in NF1 drive neurofibromatosis type I, which predisposes patients to develop a range of nervous system tumors including neurofibroma and glioma ${ }^{50}$. Although NF1 is mutated in around $10 \%$ of both pediatric and adult HGG, our findings demonstrate a near-universal mechanism of RAS/MAPK activation through AS-mediated NF1 inactivation. Signaling downstream from activated growth-factor receptors can be attenuated through a variety of feedback mechanisms, including activation of NF1 by the ribosomal S6 kinase (RSK) ${ }^{51}$. Receptors are the main RAS/MAPK pathway mutation targets in $\mathrm{HGG}^{3,4}$. As well as reducing activity toward RAS, switching isoforms to NF1-II in HGG will therefore reduce the ability of tumor cells to dampen their response to constitutive pathway activation as well as ligand-mediated signaling through other receptors.

In summary, our data demonstrate that tumors exploit nonmutagenic methods to activate oncogenic processes and, more broadly, that the absence of a mutation does not mean that oncogenic pathways will be inactive, just as the presence of a mutation in a pathway does not guarantee its activity. The decision to treat a patient with a particular targeted agent should not necessarily be based solely on mutational profiling but should also incorporate other molecular information such as splicing alterations and measures of pathway activation.

\section{Methods}

Ethical approval and patient samples. Work involving patient material was approved by the Hospital for Sick Children Research Ethics Board (\#1000055059). Written consent from a legally authorized representative (all patients in this study were under 18) was obtained to collect tissue for research in all autopsy cases and in all surgical cases collected since 2010, with explicit consent for use for nextgeneration sequencing since 2016. For surgical cases prior to 2010 or where the consent did not explicitly state the tissue would be used for next-generation sequencing, for deceased patients, waiver of consent to use the tissue for this purpose was granted by the Hospital for Sick Children Research Ethics Board.

Reagents and plasmids. Morpholinos were from GeneTools (Oregon, USA), targeted against the NF1 intron30/exon31(exon23a) and exon31(exon23a)/ intron31 junctions or a pre-designed non-targeting control. Sequences were analyzed by BLAST to confirm there were no high-specificity off-target predicted binding sites. Sequences are listed in Supplementary Table 1.

CELF4 and ELAVL3 were amplified from cDNA generated from 293T cells and cloned between the Xbal/BamHI sites of a pCDH-CMV-MCS-EF1 $\alpha$-copGFP (SystemBioscience) modified to encode a FLAG/HA tag between the BamHI/NotI sites. Primer sequences are listed in Supplementary Table 2. All plasmids were sequenced before use.

Cell culture and lentivirus generation. SF188, U87, U343, fetal NHA, and HEK293T cell lines were maintained in DMEM (VWR) supplemented with 10\% FBS (Wisent) and 1\% PenStrep (Invitrogen). SF188 cells (RRID CVCL_6948) were a kind gift from Chris Jones (Institute for Cancer Research, London). U87 (RRID CVCL_0022), U343 (RRID CVCL_4773), and HEK293T (RRID CVCL_0045) cells were from ATCC. Fetal NHA cells (T0281) were from ABM. SU-DIPG-IV (RRID CVCL IT39), SU-DIPG-VI (RRID CVCL IT40), SU-DIPG-VII, SU-DIPG-XIII (RRID CVCL_IT41), SU-DIPG-XVII, and SU-DIPG-XXXVI were a kind gift from Michele Monje (Stanford University) ${ }^{52}$ and maintained in Neurobasal $(-\mathrm{A})$ media (Invitrogen) supplemented with B27(-A; Fisher-Gibco), growth factors (humanbFGF [20 ng/ml], human-EGF (20 ng/ml), human PDGF-AA (20 ng/ml), human PDGF-BB $(20 \mathrm{ng} / \mathrm{ml})$; Gemini Bio-products) and heparin $(10 \mathrm{ng} / \mathrm{ml}$; Fisher/Stem Cell Technologies), as previously described ${ }^{52}$.

Plasmids were packaged into lentivirus by cotransfection into HEK293T cells with psPAX2 (Addgene\#12260) and pMD2.G (Addgene\#12259) using Lipofectamine 2000 (Invitrogen). The media was changed the following day and the supernatant was collected after $30 \mathrm{~h}$ and precipitated overnight with Lenti-X concentrator (Clontech) before being resuspended in Optimem (Invitrogen). Cells were transduced for $24 \mathrm{~h}$ in the presence of $10 \mu \mathrm{l} / \mathrm{ml}$ polybrene (Santa Cruz).

Cell viability assays were carried out by seeding cells in 96-well or six-well plates. The next day pladienolide B, madrasin (Cayman chemicals) or DMSO control was added and cells were incubated for 4 days. alamarBlue (Invitrogen) was added for $4 \mathrm{~h}$ and measured in a fluorescence plate reader (Molecular Devices) by exciting at $560 \mathrm{~nm}$ and measuring emission at $590 \mathrm{~nm}$, or cells were stained with trypan blue and counted. Viability was determined relative to DMSO control.

Morpholino transfections were carried out using Endo-Porter reagent (GeneTools) according to the manufacturers' instructions. Both NF1 morpholinos were simultaneously transfected each at a final concentration of $1 \mu \mathrm{M}$ (control morpholino was used at $2 \mu \mathrm{M}$ ), which allowed specific modulation of exon23a splicing without affecting NF1 expression. Cells were harvested after $48 \mathrm{~h}$ or, for differentiation experiments, $72 \mathrm{~h}$.

Differentiation assays were carried out as described ${ }^{35}$. In all, 200,000 U87 cells were seeded in six-well plates in standard media and transfected the next day with control or NF1-specific morpholinos. After $24 \mathrm{~h}$, media was changed either with standard media (control) or differentiation media (DMEM without serum, supplemented with $4 \mathrm{mM}$ sodium butyrate).

For siRNA transfections, 50,000 SU-DIPG-IV or SU-DIPG-VII cells were seeded in 24-well plates supplemented as above and transfected with either human REST smartpool or Non-targeting plus control siRNA (Dharmacon) at a final concentration of $20 \mathrm{nM}$ with DharmaFECT 1 reagent (Dharmacon) according to the manufacturers' instructions. Cells were harvested after $48 \mathrm{~h}$.

RNA extraction and analysis. Total RNA was extracted from cells or fresh-frozen tissue samples using the RNeasy kit (QIAGEN). Total RNA was reverse transcribed with 5× All-In-One RT MasterMix (Applied Biological Materials). Samples were analyzed either by reverse transcriptase PCR (RT-PCR) followed by visualization on a $2 \%$ agarose gel, or quantitative RT-PCR (qRT-PCR) with iTaq Universal SYBR green supermix (Bio-Rad) using a StepOnePlus machine (Applied Biosystems). Alternately, RNA was extracted from cells the the Quick-RNA MiniPrep Kit (Zymo Research) and cDNA synthesized with the iScript cDNA Synthesis Kit (BioRad). Samples were analyzed by qRT-PCR with a $2 \times$ SensiMix SYBR \& Fluorescein Kit (Bioline) on a Lightcycler 96 machine (Roche).

Expression from qRT-PCR was normalized using the delta-delta Ct using either beta-actin or $18 \mathrm{~S}$ housekeeping genes. Primers sequences are listed in Supplementary Table 1.

Western blotting. Extracts from cells lysed in 2x SDS lysis buffer $(20 \mathrm{mM}$ Tris $(\mathrm{pH}$ 7.4), 20 mM EDTA, 2\% SDS, 20\% glycerol) were resolved using 10-20\% SDS-PAGE gels (Invitrogen) and transferred to PVDF membranes (Amersham). Membranes were blocked and incubated overnight with primary antibody diluted in 3\% BSA in TBS with $0.1 \%$ Tween-20. After incubation with HRP-conjugated secondary antibodies (Jackson), the signal was detected with enhanced chemiluminescence 
(Pierce). Primary antibodies used were from Cell Signaling: ERK1/2 (9102, 1:1000), phospho-ERK1/2 (Thr202/204; 9101, 1:1000), COX-IV (clone 3E11; 4850, 1:1000).

Chromatin immunoprecipitation. Actively dividing SU-DIPG (IV, VII, and XIII) cells were fixed with $1 \%$ formaldehyde. Cell pellets ( 5 million per reaction) were suspended in lysis buffer (50 mM Tris- $\mathrm{HCl}(\mathrm{pH}$ 8.0), $10 \mathrm{mM}$ EDTA (pH 8.0), $1 \%$ SDS, protease inhibitors (Sigma) and chromatin sonicated with a Bioruptor Pico (Diagenode). $1 \%$ of the recovered material was saved as input and the remainder diluted fivefold in ChIP dilution buffer (16.7 mM Tris-HCl ( $\mathrm{pH} 8.0), 167 \mathrm{mM}$ $\mathrm{NaCl}, 1.2 \mathrm{mM}$ EDTA ( $\mathrm{pH} 8.0$ ), 1.1\% Triton X-100, protease inhibitors) and precleared. REST (Millipore, 07-579) or IgG (Santa Cruz, sc2027) antibodies were added overnight before incubation with protein-A beads (Millipore) and washing once each with low-salt, high-salt, and lithium chloride immune complex buffers, and twice with TE buffer. DNA was eluted with $1 \%$ SDS and $0.1 \mathrm{M} \mathrm{NaHCO}_{3}$ and cross-links reversed by incubation with $\mathrm{NaCl}$ for $4 \mathrm{~h}$ at $65^{\circ}$. DNA was purified with PCR purification kit (Zymo Research) and analyzed by qPCR with a $2 \times$ SensiMix SYBR \& Fluorescein Kit (Bioline) on a Lightcycler 96 machine (Roche).

Whole-exome/genome sequencing analysis. DNA was extracted from freshfrozen tissue samples with DNeasy kit (QIAGEN). Exome libraries were generated and sequenced at The Centre for Applied Genomics, Hospital for Sick Children. Libraries were either Ion TargetSeq Exome $50 \mathrm{Mb}$ (ThermoFisher, sequenced on Ion Proton machines and aligned to human genome hg19 with Torrent Suite Software) or TruSeq Exome (Illumina, sequenced on HiSeq 2500 machines). TruSeq exome reads were trimmed with Trimmomatic-v0.32 $2^{53}$, aligned with bwamem-v0.7. $8^{54}$, processed with the GATK suite ${ }^{55}$ and duplicate reads were marked with Picard-v2.5.0 (http://broadinstitute.github.io/picard). Variants were called with VarScan-v2.3.65 and annotated with SnpEff-v4.3k $\mathrm{k}^{57}$. To verify variants, a second algorithm was used, either GATK HaplotypeCaller for Illumina sequencing or Torrent Unified Variant Caller (ThermoFisher) for Ion Proton sequencing and key variants were further manually verified using the IGV browser. Copy number alterations were called with CNVkit-v0.8.658.

Samples were categorized into mutant groups according to the presence or absence of an alteration in a core gene of the RAS/MAPK pathway (Supplementary Data 2), the major spliceosome (HUGO group 1518; Supplementary Data 2), or the COSMIC cancer census. To be counted as mutant, either an SNV/indel with medium/high impact (missense, frameshift, nonsense, and splice site mutations) at variant-allele frequency $>0.2$ and depth $>20$, a copy number gain with $5+$ copies, or a homozygous deletion had to be present in the sample for any pathway gene. To be counted as wild-type, all genes in the pathway were required to be free of both $\mathrm{SNV} /$ indels and copy number changes. The same criteria were used for spliceosome mutations.

Where we had previously analyzed samples by WGS, WES, and SNP6.0 array to identify mutations and copy number alterations (Supplementary Data 1$)^{2}$, we used these data to infer the mutation status of the RAS pathway and spliceosome.

Mutation data for TCGA ${ }^{4,7,59}$ and CBTTC $^{11,12}$ datasets were downloaded from cBioPortal and PedcBioPortal, respectively. TCGA SNV calls were generated with Mutect and IndelLocator ${ }^{4,7,59}$, and CBTTC SNV calls come from Strelka ${ }^{11,12}$.

RNA sequencing analysis. The quality of total RNA isolated from fresh-frozen tissue was confirmed with a Bioanalyzer 2100 (Agilent). Libraries were prepared with TruSeq Stranded Total RNA Library Prep with Ribo-Zero Gold kits (Illumina, CA, USA) and sequenced at The Hospital for Sick Children with $100 \mathrm{bp}$ or $125 \mathrm{bp}$ paired-end reads on Illumina HiSeq 2500 instruments. Data were processed with the GenPipes framework ${ }^{60}$. Reads were quality trimmed with a maximum length of $100 \mathrm{bp}$ with Trimmomatic-v0.32 33 and aligned to human genome build version GRCh37-v75 using STAR-v2.5.0 in 2-pass mode ${ }^{61}$. Duplicate reads were marked with Picard-v2.5.0.

Differential expression analysis was carried out by counting expression with HTSeq $^{62}$ and testing with edgeR and DESeq ${ }^{63,64}$; only those genes with absolute fold change $>2$ and a Benjamini-Hochberg adjusted $P$ value $<0.05$ called by both edgeR and DESeq were considered to be differentially expressed. Pre-ranked gene set enrichment analysis (GSEA) ${ }^{65}$ was carried out by ranking genes with the product of their fold-change sign and the - $\log 10$ (adjusted $P$ value). t-SNE analyses were carried out with the Rtsne package in R. The RAS expression signature is from the MSigDB Hallmarks signature set and the MAPK signature from PROGENy ${ }^{66,67}$.

Transcript quantification and single-sample GSEA (ssGSEA) was carried out by aligning the trimmed reads to the transcriptome using RSEM-v1.2 ${ }^{68}$. ssGSEA was carried out using the GenePattern server after discarding genes with mean FPKM $<1$.

Splicing patterns across samples were quantified using rMATS-v4.0.116 and SUPPA $2^{18}$. Sashimi plots were drawn with rmats2sashimiplot (https://github.com/ Xinglab/rmats2sashimiplot). Events with inclusion change $>15 \%$ and $\mathrm{FDR}<0.05$ were considered to be significant. Motif enrichment was determined with the AME tool from MEME Suite-v5.4.1 using CIS-BP RNA motifs ${ }^{69,70}$. Enriched Reactome pathways were determined in Cytoscape-v3.8.2 ${ }^{71}$ using ClueGO-v2.5. $7^{72}$ with Bonferroni-adjusted $P<0.05$ and clustered according to similarity. Protein-protein interactions with experimental or database evidence were retrieved from the
STRING database $\mathrm{e}^{73}$ and visualized in Cytoscape. Pre-ranked GSEA for splicing events was carried out by ranking genes based on the - $\log 10$ (adjusted $P$ value) for the most significant event for each gene.

The amount of splicing was calculated using RSEM transcript quantification for genes with multiple transcripts. Using the R 'entropy' package, Shannon entropy $H$ for a gene $G$ with $g$ isoforms was calculated as:

$$
H(g)=-\sum_{i=1}^{g} P_{i} \cdot \log \left(P_{i}\right)
$$

where $P_{i}$ is the probability of each transcript being expressed based on transcript ratios. The median of $H$ for groups of samples was analyzed. The variability of ASE inclusion in pHGG and normal brain was assessed by plotting cumulative distribution functions of the standard deviation of ASE.

Splicing burden in COSMIC driver genes for PHGG and TCGA data were determined by identifying samples with maximal ASE inclusion change $>30 \%$ compared with normal tissue for each gene in the census.

Mouse RNA-Seq data were processed as above, except that mouse genome build version GRCm38-v83 was used.

Statistical analysis. Kaplan-Meier curves were drawn and Gehan-BreslowWilcoxon tests were carried out using GraphPad Prism 8. Unless otherwise stated, all tests are two-tailed Student's $t$ test, not assuming equal variance between samples, and were carried out in GraphPad Prism 8 or R-3.6.1

Reporting summary. Further information on research design is available in the Nature Research Reporting Summary linked to this article.

\section{Data availability}

The publicly available total RNA-Seq data from 9 pHGG and 3 normal brains are available from Gene Expression Omnibus (GEO) under accession number GSE9516923. The publicly available mouse pHGG model RNA-Seq data are available from GEO under accession numbers GSE120884 30 , GSE9516923, and GSE108364 31 . The publicly available REST ChIP-Seq data are available from GEO under accession number GSE32465 ${ }^{43}$. TFbinding sites in CELF/ELAVL gene promoters were identified with Enrichr (https:// maayanlab.cloud/Enrichr/) $/)^{74}$. RE1 motif-containing, REST-bound genes were identified from REST ChIP-Seq analysis ${ }^{44}$. Level 3 processed adult diffuse glioma RNA-Seq (RSEM quantifications of genes and isoforms), RPPA and clinical data were downloaded from The Cancer Genome Atlas/Broad Firehose (https://gdac.broadinstitute.org/), and mutations and copy number variations in the same samples were assessed with cBioPortal (https://www.cbioportal.org/). Only primary tumors were considered $4,7,59$. TCGA RNA-Seq splicing data was retrieved from TCGASpliceSeq (https:// bioinformatics.mdanderson.org/TCGASpliceSeq/singlegene.jsp) $)^{75}$. CBTTC ${ }^{11,12}$ data were retrieved from PedcBioPortal (https://pedcbioportal.org/). DNA methylation data are available in GEO under accession numbers GSE49822 and GSE55712 21,76 . Probes associated with genes contained in either the KEGG spliceosome or Reactome mRNA splicing pathway ontologies (Fig. 1b) were analyzed. Previously published data on our cohort ${ }^{2,30}$ are deposited in GEO or the European Genomics Archive (EGA); SNP6.0 data are available in GEO under accession number GSE50024; WGS data are available in EGA under accession number EGAS00001000575; WES data are available in EGA under accession numbers EGAS00001000575 and EGAD00001006450; RNA-Seq data are available in EGA under accession number EGAD00001006450. The data newly generated on our cohort is deposited with EGA; WES data are available under accession number EGAD00001008278; RNA-Seq data are available under accession EGAD00001008279. The data are available under controlled access to comply with data protection regulations, and can be accessed by application to the data access committee via C.H. (cynthia.hawkins@sickkids.ca). The remaining data are available within the Article, Supplementary Information, or Source Data file. Source data are provided with this paper.

\section{Code availability}

Custom code was not used in this study. Parameters used for data processing can be found at https://github.com/rsiddaway/NF1_paper_scripts or https://zenodo.org/record/5735134 and the corresponding DOI is as follows: https://doi.org/10.5281/zenodo.5735134.

Received: 27 May 2021; Accepted: 14 January 2022;

Published online: 31 January 2022

\section{References}

1. Khuong-Quang, D. A. et al. K27M mutation in histone H3.3 defines clinically and biologically distinct subgroups of pediatric diffuse intrinsic pontine gliomas. Acta Neuropathologica 124, 439-447 (2012).

2. Buczkowicz, P. et al. Genomic analysis of diffuse intrinsic pontine gliomas identifies three molecular subgroups and recurrent activating ACVR1 mutations. Nat. Genet. 46, 451-456 (2014). 
3. Mackay, A. et al. Integrated molecular meta-analysis of 1,000 pediatric highgrade and diffuse intrinsic pontine glioma. Cancer Cell 32, 520-537 e525 (2017).

4. Ceccarelli, M. et al. Molecular profiling reveals biologically discrete subsets and pathways of progression in diffuse glioma. Cell 164, 550-563 (2016).

5. Schwartzentruber, J. et al. Driver mutations in histone $\mathrm{H} 3.3$ and chromatin remodelling genes in paediatric glioblastoma. Nature 482, 226-231 (2012).

6. $\mathrm{Wu}, \mathrm{G}$. et al. Somatic histone $\mathrm{H} 3$ alterations in pediatric diffuse intrinsic pontine gliomas and non-brainstem glioblastomas. Nat. Genet. 44, 251-253 (2012).

7. Brennan, C. W. et al. The somatic genomic landscape of glioblastoma. Cell 155, 462-477 (2013).

8. Zarghooni, M. et al. Whole-genome profiling of pediatric diffuse intrinsic pontine gliomas highlights platelet-derived growth factor receptor alpha and poly (ADP-ribose) polymerase as potential therapeutic targets. J. Clin. Oncol 28, 1337-1344 (2010).

9. Sanchez-Vega, F. et al. Oncogenic signaling pathways in The Cancer Genome Atlas. Cell 173, 321-337 e310 (2018).

10. Kahles, A. et al. Comprehensive analysis of alternative splicing across tumors from 8,705 patients. Cancer Cell 34, 211-224 e216 (2018).

11. Ijaz, H. et al. Pediatric high-grade glioma resources from the Children's Brain Tumor Tissue Consortium. Neuro-Oncol. 22, 163-165 (2020).

12. Petralia, F. et al. Integrated proteogenomic characterization across major histological types of pediatric brain cancer. Cell 183, 1962-1985 e1931 (2020).

13. Chesnais, V. et al. Spliceosome mutations in myelodysplastic syndromes and chronic myelomonocytic leukemia. Oncotarget 3, 1284-1293 (2012).

14. Shuai, S. et al. The U1 spliceosomal RNA is recurrently mutated in multiple cancers. Nature 574, 712-716 (2019)

15. Suzuki, H. et al. Recurrent noncoding U1 snRNA mutations drive cryptic splicing in SHH medulloblastoma. Nature 574, 707-711 (2019).

16. Shen, S. et al. rMATS: robust and flexible detection of differential alternative splicing from replicate RNA-Seq data. Proc. Natl Acad. Sci. USA 111 E5593-E5601 (2014).

17. Ritchie, W., Granjeaud, S., Puthier, D. \& Gautheret, D. Entropy measures quantify global splicing disorders in cancer. PLoS Comput. Biol. 4, e1000011 (2008).

18. Trincado, J. L. et al. SUPPA2: fast, accurate, and uncertainty-aware differential splicing analysis across multiple conditions. Genome Biol. 19, 40 (2018).

19. Chen, X. et al. Systematic profiling of alternative mRNA splicing signature for predicting glioblastoma prognosis. Front. Oncol. 9, 928 (2019).

20. Sondka, Z. et al. The COSMIC Cancer Gene Census: describing genetic dysfunction across all human cancers. Nat. Rev. Cancer 18, 696-705 (2018).

21. Bender, S. et al. Reduced H3K27me3 and DNA hypomethylation are major drivers of gene expression in $\mathrm{K} 27 \mathrm{M}$ mutant pediatric high-grade gliomas. Cancer Cell 24, 660-672 (2013).

22. Batsche, E., Yaniv, M. \& Muchardt, C. The human SWI/SNF subunit Brm is a regulator of alternative splicing. Nat. Struct. Mol. Biol. 13, 22-29 (2006).

23. Pathania, M. et al. H3.3(K27M) cooperates with Trp53 loss and PDGFRA gain in mouse embryonic neural progenitor cells to induce invasive high-grade gliomas. Cancer Cell 32, 684-700 e689 (2017).

24. Marquette, A., Andre, J., Bagot, M., Bensussan, A. \& Dumaz, N. ERK and PDE4 cooperate to induce RAF isoform switching in melanoma. Nat. Struct. Mol. Biol. 18, 584-591 (2011).

25. Hallberg, B., Ashcroft, M., Loeb, D. M., Kaplan, D. R. \& Downward, J. Nerve growth factor induced stimulation of Ras requires Trk interaction with Shc but does not involve phosphoinositide 3-OH kinase. Oncogene 17, 691-697 (1998)

26. Shi, E. et al. Control of fibroblast growth factor receptor kinase signal transduction by heterodimerization of combinatorial splice variants. Mol. Cell. Biol. 13, 3907-3918 (1993).

27. Yamaguchi, F., Saya, H., Bruner, J. M. \& Morrison, R. S. Differential expression of two fibroblast growth factor-receptor genes is associated with malignant progression in human astrocytomas. Proc. Natl Acad. Sci. USA 91, 484-488 (1994).

28. Andersen, L. B. et al. A conserved alternative splice in the von Recklinghausen neurofibromatosis (NF1) gene produces two neurofibromin isoforms, both of which have GTPase-activating protein activity. Mol. Cell. Biol. 13, 487-495 (1993)

29. Scheffzek, K. et al. Structural analysis of the GAP-related domain from neurofibromin and its implications. EMBO J. 17, 4313-4327 (1998)

30. Pajovic, S. et al. Epigenetic activation of a RAS/MYC axis in H3.3K27Mdriven cancer. Nat. Commun. 11, 6216 (2020).

31. Larson, J. D. et al. Histone H3.3 K27M accelerates spontaneous brainstem glioma and drives restricted changes in bivalent gene expression. Cancer Cell 35, 140-155 e147 (2019).

32. Hinman, M. N., Sharma, A., Luo, G. \& Lou, H. Neurofibromatosis type 1 alternative splicing is a key regulator of Ras signaling in neurons. Mol. Cell. Biol. 34, 2188-2197 (2014).
33. Nguyen, H. T. et al. Neurofibromatosis type 1 alternative splicing is a key regulator of Ras/ERK signaling and learning behaviors in mice. Hum. Mol. Genet. 26, 3797-3807 (2017).

34. Biayna, J. et al. Using antisense oligonucleotides for the physiological modulation of the alternative splicing of NF1 exon 23a during PC12 neuronal differentiation. Sci. Rep. 11, 3661 (2021).

35. Liu, H. et al. Differentiation of human glioblastoma U87 cells into cholinergic neuron. Neurosci. Lett. 704, 1-7 (2019).

36. Dai, N. et al. IGF2 mRNA binding protein-2 is a tumor promoter that drives cancer proliferation through its client mRNAs IGF2 and HMGA1. eLife 6 https://doi.org/10.7554/eLife.27155 (2017).

37. Barron, V. A., Zhu, H., Hinman, M. N., Ladd, A. N. \& Lou, H. The neurofibromatosis type I pre-mRNA is a novel target of CELF proteinmediated splicing regulation. Nucleic Acids Res. 38, 253-264 (2010).

38. Zhu, H., Hinman, M. N., Hasman, R. A., Mehta, P. \& Lou, H. Regulation of neuron-specific alternative splicing of neurofibromatosis type 1 pre-mRNA. Mol. Cell. Biol. 28, 1240-1251 (2008).

39. Conti, L. et al. REST controls self-renewal and tumorigenic competence of human glioblastoma cells. PLoS ONE 7, e38486 (2012).

40. Kamal, M. M. et al. REST regulates oncogenic properties of glioblastoma stem cells. Stem Cells 30, 405-414 (2012).

41. Liang, J. et al. An expression based REST signature predicts patient survival and therapeutic response for glioblastoma multiforme. Sci. Rep. 6, 34556 (2016).

42. Shaik, S. et al. REST upregulates gremlin to modulate diffuse intrinsic pontine glioma vasculature. Oncotarget 9, 5233-5250 (2018).

43. Gertz, J. et al. Distinct properties of cell-type-specific and shared transcription factor binding sites. Mol. Cell 52, 25-36 (2013).

44. Johnson, D. S., Mortazavi, A., Myers, R. M. \& Wold, B. Genome-wide mapping of in vivo protein-DNA interactions. Science 316, 1497-1502 (2007).

45. Sebestyen, E. et al. Large-scale analysis of genome and transcriptome alterations in multiple tumors unveils novel cancer-relevant splicing networks. Genome Res. 26, 732-744 (2016).

46. Seiler, M. et al. Somatic mutational landscape of splicing factor genes and their functional consequences across 33 cancer types. Cell Rep. 23, 282-296 e284 (2018).

47. Zhu, L. Y., Zhu, Y. R., Dai, D. J., Wang, X. \& Jin, H. C. Epigenetic regulation of alternative splicing. Am. J. Cancer Res. 8, 2346-2358 (2018).

48. Suzuki, Y., Suzuki, H., Kayama, T., Yoshimoto, T. \& Shibahara, S. Brain tumors predominantly express the neurofibromatosis type 1 gene transcripts containing the 63 base insert in the region coding for GTPase activating protein-related domain. Biochemical Biophysical Res. Commun. 181, 955-961 (1991).

49. Scheurlen, W. G. \& Senf, L. Analysis of the GAP-related domain of the neurofibromatosis type 1 (NF1) gene in childhood brain tumors. Int. J. Cancer 64, 234-238 (1995)

50. Bader, J. L. Neurofibromatosis and cancer. Ann. N. Y. Acad. Sci. 486, 57-65 (1986)

51. Hennig, A. et al. Feedback activation of neurofibromin terminates growth factor-induced Ras activation. Cell Commun. Signal.: CCS 14, 5 (2016).

52. Grasso, C. S. et al. Functionally defined therapeutic targets in diffuse intrinsic pontine glioma. Nat. Med. 21, 555-559 (2015).

53. Bolger, A. M., Lohse, M. \& Usadel, B. Trimmomatic: a flexible trimmer for Illumina sequence data. Bioinformatics 30, 2114-2120 (2014).

54. Li, H. \& Durbin, R. Fast and accurate short read alignment with BurrowsWheeler transform. Bioinformatics 25, 1754-1760 (2009).

55. Van der Auwera, G. A. et al. From FastQ data to high confidence variant calls the Genome Analysis Toolkit best practices pipeline. Curr. Protoc. Bioinforma. 43, $111011-111033$ (2013).

56. Koboldt, D. C. et al. VarScan 2: somatic mutation and copy number alteration discovery in cancer by exome sequencing. Genome Res. 22, 568-576 (2012).

57. Cingolani, P. et al. A program for annotating and predicting the effects of single nucleotide polymorphisms, SnpEff: SNPs in the genome of Drosophila melanogaster strain w1118; iso-2; iso-3. Fly 6, 80-92 (2012).

58. Talevich, E., Shain, A. H., Botton, T. \& Bastian, B. C. CNVkit: genome-wide copy number detection and visualization from targeted DNA sequencing. PLoS Comput. Biol. 12, e1004873 (2016).

59. Cancer Genome Atlas Research, N. et al. Comprehensive, integrative genomic analysis of diffuse lower-grade gliomas. N. Engl. J. Med. 372, 2481-2498 (2015).

60. Bourgey, M. et al. GenPipes: an open-source framework for distributed and scalable genomic analyses. GigaScience 8, https://doi.org/10.1093/gigascience/ giz037 (2019).

61. Dobin, A. et al. STAR: ultrafast universal RNA-seq aligner. Bioinformatics 29, 15-21 (2013).

62. Anders, S., Pyl, P. T. \& Huber, W. HTSeq-a Python framework to work with high-throughput sequencing data. Bioinformatics 31, 166-169 (2015). 
63. Robinson, M. D., McCarthy, D. J. \& Smyth, G. K. edgeR: a Bioconductor package for differential expression analysis of digital gene expression data. Bioinformatics 26, 139-140 (2010).

64. Anders, S. \& Huber, W. Differential expression analysis for sequence count data. Genome Biol. 11, R106 (2010).

65. Subramanian, A. et al. Gene set enrichment analysis: a knowledge-based approach for interpreting genome-wide expression profiles. Proc. Natl Acad. Sci. USA 102, 15545-15550 (2005).

66. Liberzon, A. et al. The Molecular Signatures Database (MSigDB) hallmark gene set collection. Cell Syst. 1, 417-425 (2015).

67. Schubert, M. et al. Perturbation-response genes reveal signaling footprints in cancer gene expression. Nat. Commun. 9, 20 (2018).

68. Li, B. \& Dewey, C. N. RSEM: accurate transcript quantification from RNA-Seq data with or without a reference genome. BMC Bioinforma. 12, 323 (2011).

69. Bailey, T. L., Johnson, J., Grant, C. E. \& Noble, W. S. The MEME Suite. Nucleic Acids Res. 43, W39-W49 (2015).

70. Ray, D. et al. A compendium of RNA-binding motifs for decoding gene regulation. Nature 499, 172-177 (2013).

71. Shannon, P. et al. Cytoscape: a software environment for integrated models of biomolecular interaction networks. Genome Res. 13, 2498-2504 (2003).

72. Bindea, G. et al. ClueGO: a Cytoscape plug-in to decipher functionally grouped gene ontology and pathway annotation networks. Bioinformatics 25, 1091-1093 (2009).

73. Szklarczyk, D. et al. STRING v11: protein-protein association networks with increased coverage, supporting functional discovery in genome-wide experimental datasets. Nucleic Acids Res. 47, D607-D613 (2019).

74. Kuleshov, M. V. et al. Enrichr: a comprehensive gene set enrichment analysis web server 2016 update. Nucleic Acids Res. 44, W90-W97 (2016)

75. Ryan, M. et al. TCGASpliceSeq a compendium of alternative mRNA splicing in cancer. Nucleic Acids Res. 44, D1018-D1022 (2016).

76. Fontebasso, A. M. et al. Recurrent somatic mutations in ACVR1 in pediatric midline high-grade astrocytoma. Nat. Genet. 46, 462-466 (2014).

\section{Acknowledgements}

We thank the Hawkins lab for their insight during this work. C.H. was funded by the Canadian Cancer Society Research Institute (702296 and 706160), Canadian Institutes of Health Research (PJT-156407), ChadTough Foundation, DIPG Collaborative, and Meagan's Walk; J.N. was funded by The Gilbert Family Foundation. The results shown here are in part based upon data generated by the TCGA Research Network: https:// www.cancer.gov/tcga.

\section{Author contributions}

Conceptualization: R.S. and C.H. Methodology: R.S., M.B., A.R., and C.H. Formal analysis: R.S., A.R., A.K.A.V., and P.P. Investigation: R.S., S.M., J.S., T.D., A.K.A.V., S.R., and S.P. Visualization: R.S., A.K.A.V., and P.P. Resources: J.N. and O.B. Writing: R.S. and C.H. Supervision: M.B., V.G., and C.H. Funding acquisition: C.H.

\section{Competing interests}

The authors declare no competing interests.

\section{Additional information}

Supplementary information The online version contains supplementary material available at https://doi.org/10.1038/s41467-022-28253-4.

Correspondence and requests for materials should be addressed to Cynthia Hawkins.

Peer review information Nature Communications thanks the anonymous reviewers for their contribution to the peer review of this work.

Reprints and permission information is available at http://www.nature.com/reprints

Publisher's note Springer Nature remains neutral with regard to jurisdictional claims in published maps and institutional affiliations.

(c) (i) Open Access This article is licensed under a Creative Commons Attribution 4.0 International License, which permits use, sharing, adaptation, distribution and reproduction in any medium or format, as long as you give appropriate credit to the original author(s) and the source, provide a link to the Creative Commons license, and indicate if changes were made. The images or other third party material in this article are included in the article's Creative Commons license, unless indicated otherwise in a credit line to the material. If material is not included in the article's Creative Commons license and your intended use is not permitted by statutory regulation or exceeds the permitted use, you will need to obtain permission directly from the copyright holder. To view a copy of this license, visit http://creativecommons.org/ licenses/by/4.0/.

(C) The Author(s) 2022 